\title{
APPLYING COORDINATE PRODUCTS TO THE TOPOLOGICAL IDENTIFICATION OF NORMED SPACES
}

\author{
ROBERT CAUTY AND TADEUSZ DOBROWOLSKI
}

\begin{abstract}
Using the $l^{2}$-products we find pre-Hilbert spaces that are absorbing sets for all Borelian classes of order $\alpha \geq 1$. We also show that the following spaces are homeomorphic to $\Sigma^{\infty}$, the countable product of the space $\Sigma=$ $\left\{\left(x_{n}\right) \in R^{\infty}:\left(x_{n}\right)\right.$ is bounded $\}$ :

(1) every coordinate product $\prod_{C} H_{n}$ of normed spaces $H_{n}$ in the sense of a Banach space $C$, where each $H_{n}$ is an absolute $F_{\sigma \delta}$-set and infinitely many of the $H_{n}$ 's are $Z_{\sigma}$-spaces,

(2) every function space $\widetilde{L}^{p}=\bigcap_{p^{\prime}<p} L^{p^{\prime}}$ with the $L^{q}$-topology, $0<q<$ $p \leq \infty$,

(3) every sequence space $\widetilde{l}^{p}=\bigcap_{p<p^{\prime}} l^{p^{\prime}}$ with the $l^{q}$-topology, $0 \leq p<q<$ $\infty$.

We also note that each additive and multiplicative Borelian class of order $\alpha \geq 2$, each projective class, and the class of nonprojective spaces contain uncountably many topologically different pre-Hilbert spaces which are $Z_{\sigma}$-spaces.
\end{abstract}

\section{INTRODUCTION}

We are interested in the topological classification of noncomplete normed linear spaces. The main tool in this area is the method of absorbing sets discovered and applied in the $\sigma$-compact case by Anderson and Bessaga and Petczyński (see [2]). Absorbing sets which are not necessarily $\sigma$-closed in a considered copy $s$ of $l^{2}$ were developed by Bestvina and Mogilski [4]. A disadvantage of the approach presented in [4] was that two homeomorphic absorbing sets in $s$ might not have been relatively homeomorphic. The difficulty was overcome in [7] due to replacing the strong universality property by its relative version (see Theorem 2.2). We construct linear subspaces $F_{\alpha}, \alpha \geq 1$ (respectively, $G_{\alpha}$, $\alpha \geq 2$ ) of $l^{2}$ that are absorbing sets for the additive Borelian class $\mathscr{A}_{\alpha}$ (respectively, the multiplicative Borelian class $\left.\mathscr{M}_{\alpha}\right)$ and such that the pair $\left(l^{2}, F_{\alpha}\right)$ (respectively, $\left.\left(l^{2}, G_{\alpha}\right)\right)$ is strongly $\left(\mathscr{M}_{1}, \mathscr{A}_{\alpha}\right)$-universal (respectively, $\left(\mathscr{M}_{1}, \mathscr{M}_{\alpha}\right)$ universal). Applying Theorem $2.2,\left(l^{2}, F_{\alpha}\right)$ and $\left(l^{2}, G_{\alpha}\right)$ are homeomorphic to $\left(s, \Lambda_{\alpha}\right)$ and $\left(s, \Omega_{\alpha}\right)$, respectively, where $\Lambda_{\alpha}$ and $\Omega_{\alpha}$ are absorbing sets in $s$ constructed in [4].

One may guess that $F_{\alpha}$ (respectively, $G_{\alpha}$ ) is the weak $l^{2}$-product $\sum_{l^{2}} H_{n}$ (respectively, the $l^{2}$-product $\prod_{l^{2}} H_{n}$ ) of pre-Hilbert spaces $H_{n}$ that contain a

Received by the editors October 11, 1990.

1980 Mathematics Subject Classification (1985 Revision). Primary 57N17.

Key words and phrases. Coordinate product, pre-Hilbert space, strong $(\mathscr{K}, \mathscr{L})$-universality, absorbing set, absolute $F_{\sigma \delta}$-set. 
closed copy of $\Lambda_{\alpha}$ (respectively, $\Omega_{\alpha}$ ). The crucial step is to show that $\sum_{l^{2}} H_{n}$ and $\prod_{l^{2}} H_{n}$ are strongly $\mathscr{A}_{\alpha^{-}}$and $\mathscr{M}_{\alpha}$-universal, respectively. Actually, we are able to verify the strong $(\mathscr{K}, \mathscr{L})$-universality property of an arbitrary normed coordinate product pair $\left(\prod_{C} E_{n}, \sum_{C} H_{n}\right)$ provided each element of $(\mathscr{K}, \mathscr{L})$ admits a relative closed embedding into every $\left(E_{n}, H_{n}\right)$ (see Proposition 3.1). A version of 3.1 for cartesian products was earlier applied $[11,13,12]$ in order to identify some function and sequence spaces that are homeomorphic to $\Omega_{2}=$ $\Sigma^{\infty}$. Applying 3.1 (and its variations), we show that several absolute $F_{\sigma \delta}$-spaces that underlie a "product" structure are homeomorphic to $\Omega_{2}$. In particular, we prove that every normed coordinate product $\prod_{C} H_{n}, C$ being a Banach space, is homeomorphic to $\Omega_{2}$ provided each $H_{n} \in \mathscr{M}_{2}$ and infinitely many of the $H_{n}$ 's are $Z_{\sigma}$-spaces. Another application concerns the function space $\widetilde{L}^{p}=\bigcap_{p^{\prime}<p} L^{p^{\prime}}$ in the $L^{q}$-topology $(q<p)$ and the sequence space $\widetilde{l}^{p}=$ $\bigcap_{p<p^{\prime}} l^{p^{\prime}}$ in the $l^{q}$-topology $(p<q)$. We prove that $\widetilde{L}^{p}, 0<q<p \leq \infty$, and $\widetilde{l}^{p}, 0 \leq p<q<\infty$, are homeomorphic to $\Omega_{2}$. Actually, we show that the pairs $\left(L^{q}, \widetilde{L}^{p}\right),\left(l^{q}, \widetilde{l}^{p}\right)$, and $\left(s, \Omega_{2}\right)$ are homeomorphic. The fact that the space $\widetilde{L}^{p}$ considered as a subspace of $L^{0}$ (of all measurable functions with the topology of convergence in measure) and the space $\widetilde{l}^{p}$ as a subspace of $R^{\infty}$ are homeomorphic to $\Omega_{2}$ was previously obtained in [13]. Let us note that dealing with these different topologies on $\widetilde{L}^{p}$ (same for $\widetilde{l}^{p}$ ) the natural linear map $\Psi: L^{0} \rightarrow\left(L^{0}\right)^{\infty}$ is employed. In the present paper $\Psi$ is considered as a linear isomorphism of $L^{1}$ onto $\prod_{l^{1}} L^{1}$ with the following key property:

$$
\Psi\left(\widetilde{L}^{p}\right) \cap \sum_{l^{1}} L^{1}=\sum_{l^{1}} \tilde{L}^{p} .
$$

In the last section we provide some examples of pre-Hilbert spaces with rather mysterious topological structure. They all are of the form

$$
Y(A) \times F_{\alpha} \text { and } Y(A) \times G_{\alpha},
$$

where $Y(A)$ is the linear span of a linearly independent subset $A$ in $l^{2}$. In particular, we show that every projective class $\mathscr{P}_{n} \backslash \bigcup_{k<n} \mathscr{P}_{k}, n \geq 1$, contains uncountably many nonhomeomorphic pre-Hilbert spaces. The same is true for the class of spaces which are nonprojective. We observe that the argument of Henderson and Petczyński [2] showing that there are uncountably many $\sigma$-compact pre-Hilbert spaces applies (after a minor change) to produce uncountably many nonhomeomorphic pre-Hilbert spaces in each class $\mathscr{A}_{\alpha} \backslash \mathscr{M}_{\alpha}$ and $\mathscr{M}_{\alpha} \backslash \mathscr{A}_{\alpha}$ for $\alpha \geq 2$.

The results of $\S 3$ will be applied to construct absorbing sets for all projective classes in a forthcoming paper by the first named author.

The authors wish to note that J. Dijkstra and J. Mogilski have recently obtained the same results concerning $\widetilde{L}^{p}$ - and $\widetilde{l}^{p}$-spaces [10].

Convention. All spaces considered are separable and metrizable. Maps are continuous functions.

\section{Preliminaries}

Let us recall that a closed subset $A$ of a space $X$ is a $Z$-set (respectively, a strong $Z$-set) if for every open cover $\mathscr{U}$ of $X$ there exists a $\mathscr{U}$-close to the 
identity map $f: X \rightarrow X$ such that $f(X) \cap A=\varnothing$ (respectively, $\operatorname{cl}(f(X)) \cap A=$ $\varnothing)$. A space which is a countable union of $Z$-sets is called a $Z_{\sigma}$-space. Note that every $Z_{\sigma}$-space is of the first category. In the case where $X$ is an absolute neighborhood retract a closed set $A$ is a $Z$-set iff given $n$ every map of the $n$ dimensional cube $I^{n}$ into $X$ can be approximated by maps into $X \backslash A$. Every not necessarily closed set $A$ satisfying the above condition is called locally homotopy negligible in $X$ (see [17]).

Fix a pair of spaces $(K, L)$, i.e., $L \subseteq K$. We say that a pair of spaces $(X, Y)$ is strongly $(K, L)$-universal if, for every closed subset $D$ of $K$, every map $f: K \rightarrow X$ whose restriction to $D$ is a $Z$-embedding (i.e., $f \mid D$ is an embedding and $f(D)$ is a $Z$-set in $X$ ) and satisfies the condition

$$
(f \mid D)^{-1}(Y)=D \cap L,
$$

and every open cover $\mathscr{U}$ of $X$, there exists a $Z$-embedding $g: K \rightarrow X$ which is $\mathscr{U}$-close to $f$ and satisfies the conditions

$$
g|D=f| D \quad \text { and } \quad g^{-1}(Y)=L .
$$

We find it convenient to formulate the following technical fact concerning the strong $(K, L)$-universality (cf. [4, Proposition 2.2]).

Proposition 2.1. Let an absolute neighborhood retract $X$, its subsets $Y \subseteq Y^{\prime}$, and a pair of spaces $(K, L)$ satisfy the following conditions:

(i) every $Z$-set in $X$ is a strong $Z$-set,

(ii) $X \backslash Y$ is locally homotopy negligible in $X$,

(iii) $Y^{\prime}$ is locally homotopy negligible in $X$,

(iv) given open subsets $U$ of $K$ and $V$ of $X$, a map $f: K \rightarrow X$ with $f(U) \subset V \cap Y$ and $f(K \backslash U) \subset X \backslash V$, and an open cover $\mathscr{V}$ of $V$, there exists a closed embedding $g: U \rightarrow V$ which is $\mathscr{V}$-close to $f \mid U$ and satisfies $g(U) \subset Y^{\prime}$ and $g^{-1}(V \cap Y)=L \cap U$.

Then for every $Z \subseteq X$ with $Z \cap Y^{\prime}=Y$, the pair $(X, Z)$ is strongly $(K, L)$ universal.

Before we give a proof of 2.1 we recall that $f: K \rightarrow X$ is closed over a set $A \subset X$ if for every $a \in A$ and every neighborhood $U$ of $f^{-1}(\{a\})$ there exists a neighborhood $V$ of $a$ such that $f^{-1}(V) \subset U$ (see [4]).

Proof of 2.1. Let $D$ be a closed subset of $K$ and let $\bar{f}: K \rightarrow X$ be a map such that $\bar{f} \mid D$ is a $Z$-embedding satisfying $(\bar{f} \mid D)^{-1}(Z)=D \cap L$. Since $\bar{f}(D)$ is a strong $Z$-set in $X$ and $X \backslash Y$ is locally homotopy negligible in $X$, we can apply [4, Lemma $1.1 ; 17$, Theorem 2.4] to approximate $\bar{f}$ by $f$ such that

(1) $\bar{f}|D=f| D$,

(2) $f$ is closed over $\bar{f}(D)$,

(3) $f(K \backslash D) \subset Y \backslash f(D)$.

Set $U=K \backslash D$ and $V=X \backslash f(D)$. Let $\mathscr{U}$ be an open cover of $X$. Fix a metric $d$ on $X$ and choose an open cover $\mathscr{V}$ of $V$ which is inscribed in $\mathscr{U}$ and such that

(4) for every element $W$ of $\mathscr{V}, \operatorname{diam}(W)<\operatorname{dist}(W, X \backslash V)$.

By our assumption, there exists a closed embedding $g: U \rightarrow V$ which is $\mathscr{V}$ close to $f \mid U$ and such that $g^{-1}(V \cap Y)=U \cap L$ and $g(U) \subset Y^{\prime}$. By (4), $g$ 
can be continuously extended by $\bar{f}=f$ over $D$ to a one-to-one map which is $\mathscr{U}$-close to $f$. Denote this extension also by $g$. We have $g^{-1}(V \cap Y)=$ $U \cap L$ and consequently $g(L) \subset Z$. Moreover, if $g(x) \in Z$ and $x \notin D$ then $g(x) \in Z \cap Y^{\prime}=Y$. This, together with $(\bar{f} \mid D)^{-1}(Z)=D \cap L$, yields $g^{-1}(Z)=L$. To show that $g: K \rightarrow X$ is a closed embedding, let $\left\{g\left(x_{n}\right)\right\}_{n=1}^{\infty}$ converge to $y \in X$. If $y \in \bar{f}(D)$ then, by (4), $\left\{f\left(x_{n}\right)\right\}_{n=1}^{\infty}$ converges to $y$ and consequently, by (2), $\left\{x_{n}\right\}_{n=1}^{\infty}$ converges to $f^{-1}(y)$. Otherwise, $y \in V$ and $\left\{x_{n}\right\}_{n=1}^{\infty}$ converges to $g^{-1}(y)$. Since $g(K) \subset \bar{f}(D) \cup Y^{\prime}$, the union of a $Z$-set and a locally homotopy negligible set, $g(K)$, is a $Z$-set in $X$.

Let $\mathscr{K}$ and $\mathscr{L}$ be classes of spaces. We write $(K, L) \in(\mathscr{K}, \mathscr{L})$ provided $K \in \mathscr{K}$ and $L \in \mathscr{L}$. A pair of spaces $(X, Y)$ is said to be strongly $(\mathscr{K}, \mathscr{L})$ universal if $(X, Y)$ is strongly $(K, L)$-universal for every pair $(K, L) \in$ $(\mathscr{K}, \mathscr{L})$. This concept was introduced in [6]. If the pair $(Y, Y)$ is strongly $(L, L)$-universal for every $L \in \mathscr{L}$ then, according to [4], $Y$ is strongly $\mathscr{L}$ universal.

In what follows, $\mathscr{L}$ will satisfy the following conditions:

(a) if $L$ and $L^{\prime}$ are homeomorphic and $L \in \mathscr{L}$, then $L^{\prime} \in \mathscr{L}$,

(b) if a space $L$ is a union of its two closed subspaces which belong to $\mathscr{L}$, then $L \in \mathscr{L}$,

(c) every closed subset of an element of $\mathscr{L}$ belongs to $\mathscr{L}$.

The following fact proved in [7] extends the uniqueness theorem for absorbing sets discovered by Anderson and Bessaga and Pelczyński (see [2]).

Theorem 2.2 [7, Theorem 2.1]. Let $X$ be a topological copy of $l^{2}$ and let $Y_{1}$ and $Y_{2}$ be two subsets of $X$. Assume that both $Y=Y_{1}$ and $Y_{2}$ satisfy the following conditions:

(i) $X \backslash Y$ is locally homotopy negligible in $X$,

(ii) $Y$ is a $Z_{\sigma}$-space,

(iii) $Y$ is a countable union of closed sets that are elements of $\mathscr{L}$,

(iv) $(X, Y)$ is strongly $(\mathscr{M}, \mathscr{L})$-universal, where $\mathscr{M}$ is the class of completely metrizable spaces.

Then, for every open cover $\mathscr{U}$ of $X$, there exists a $\mathscr{U}$-close to the identity homeomorphism of $\left(X, Y_{1}\right)$ onto $\left(X, Y_{2}\right)$.

Every subset $Y$ of $X$ which is strongly $\mathscr{L}$-universal and fulfils (i)-(iii) is called an $\mathscr{L}$-absorbing set in $X$. In [4], it was shown that two $\mathscr{L}$-absorbing sets in a copy of $l^{2}$ are homeomorphic. Theorem 2.2 may be rephrased in its weaker form as follows: two $\mathscr{L}$-absorbing sets in a copy of $l^{2}$ are relatively homeomorphic provided they are strongly $(\mathscr{M}, \mathscr{L})$-universal.

\section{STRONG UNIVERSALITY IN PRODUCTS}

Let $C$ be a normed countable coordinate space (briefly, a normed coordinate space), i.e., $C=\left(C,\|\cdot\|_{C}\right)$ is a normed linear space of real sequences such that

$\left(\mathrm{c}_{1}\right)$ for every bounded sequence $\lambda=\left(\lambda_{n}\right)$ and every $c=\left(c_{n}\right) \in C$, we have $\lambda \cdot c=\left(\lambda_{n} c_{n}\right) \in C$ and $\|\lambda \cdot c\|_{C} \leq\|\lambda\|_{\infty}\|c\|_{C}$, where $\|\lambda\|_{\infty}=\sup _{n \geq 1}\left|\lambda_{n}\right|$,

$\left(c_{2}\right)$ for every $\varepsilon>0$ and every $\left(c_{n}\right) \in C$ there exists $k$ such that

$$
\left\|\left(0, \ldots, 0, c_{k}, c_{k+1}, \ldots\right)\right\|_{C}<\varepsilon
$$


$\left(c_{3}\right)$ each unit vector $u_{n}=\left(\delta_{n}^{i}\right)$ belongs to $C$.

We took the notion of a normed coordinate space from [1] (see also [16]) where the following equivalent condition replaces $\left(c_{3}\right): C$ is contained in no hyperplane $\left\{\left(c_{n}\right): c_{k}=0\right\}, k \geq 1$. (For examples of normed coordinate spaces, see [1].) Note that $C$ contains all eventually zero sequences $C_{0}$. Later on we have to assume that $C \backslash C_{0} \neq \varnothing$. This, of course, is the case if $C$ is a Banach space.

Let $\left\{\left(E_{n},\|\cdot\|_{n}\right)\right\}_{n=1}^{\infty}$ be a sequence of normed linear spaces. We consider the linear spaces

$$
\prod_{C} E_{n}=\left\{\left(y_{n}\right) \in \prod_{n=1}^{\infty} E_{n}:\left(\left\|y_{n}\right\|_{n}\right) \in C\right\}
$$

and

$$
\sum_{C} E_{n}=\left\{\left(y_{n}\right) \in \prod_{n=1}^{\infty} E_{n}: y_{n}=0 \text { for almost all } n\right\}
$$

which are both equipped with the norm $\left\|\left(y_{n}\right)\right\|\|=\|\left(\left\|y_{n}\right\|_{n}\right) \|_{C}$. These spaces are called, respectively, the normed coordinate product (of the $E_{n}$ 's in the sense of $C$ ) and the weak normed coordinate product (briefly, $C$-product and weak $C$-product of the $E_{n}$ 's). For $y=\left(y_{n}\right) \in \prod_{C} E_{n}$ and $k \geq 1$, we write $r_{k}(y)=\left(0, \ldots, y_{k}, y_{k+1}, \ldots\right), s_{k}(y)=y-r_{k}(y)$, and $\pi_{k}(y)=y_{k}$. Identifying $E_{n}$ with the natural subspace of $\prod_{C} E_{n}$, we have

(A) $\left|\left\|s_{k}(y)\right\|\right| \leq|\|y \mid\|$,

(B) $\quad\left\|\pi_{k}(y)\right\|\left|\leq\left\|\left|\left\|r_{k}(y)\right\|\right| \leq\right\|\right|\|y\| \|$,

(C) $\lim \left\|\mid r_{k}(y)\right\| \|=0$,

for every $k \geq 1$ and $y \in \prod_{C} E_{n}$.

We now give the main result of this section.

Proposition 3.1. Let $\left\{\left(E_{n}, H_{n}\right)\right\}_{n=1}^{\infty}$ be a sequence of pairs of nontrivial normed linear spaces with each $H_{n}$ dense in $E_{n}$ and let $C$ be a normed coordinate space that contains an element with infinitely many nonzero terms. Fix a pair of spaces $(K, L)$ and assume that for every $n \geq 1$ there exists a bounded closed embedding $\psi_{n}: K \rightarrow E_{n}$ with $\psi^{-1}\left(H_{n}\right)=L$. Then, for every $Z \subseteq E=\prod_{C} E_{n}$ with $Z \cap \sum_{C} E_{n}=\sum_{C} H_{n}$, the pair $(E, Z)$ is strongly $(K, L)$-universal.

We shall make use of the next two lemmas.

Lemma 3.2. There exists a homotopy

$$
\Phi=\left(\Phi_{n}\right):\left(E \times[0,1], \sum_{C} H_{n} \times[0,1]\right) \rightarrow\left(E, \sum_{C} H_{n}\right)
$$

satisfying the following conditions:

(i) $\Phi(\cdot, 0)=$ id

(ii) if $n \geq \frac{1}{t}+2$, then $\Phi_{n}(y, t)=0$,

(iii) if for some sequence $\left\{\left(y(i), t_{i}\right)\right\}_{i=1}^{\infty} \subset E \times[0,1]$ with $\lim t_{i}=0$ there exists $y \in E$ such that $\lim \Phi\left(y(i), t_{i}\right)=y$, then $\lim y(i)=y$.

Lemma 3.3. There exists a one-to-one map

$$
\varphi: K \times(0,1] \rightarrow E
$$


satisfying the following conditions:

(iv) $\varphi^{-1}\left(\sum_{C} H_{n}\right)=L \times(0,1]$,

(v) $\|\varphi(x, t)\| \leq \leq t$ for all $(x, t) \in K \times(0,1]$,

(vi) if $x \in K$ and $\frac{1}{n+1}<t \leq \frac{1}{n}$, then $\pi_{n+2} \varphi(x, t) \neq 0$ while $\pi_{k} \varphi(x, t)=0$ for all $k<n$ and $k \geq n+4$,

(vii) if the sequence $\left\{\varphi\left(x_{i}, t_{i}\right)\right\}_{i=1}^{\infty}$ converges in $E,\left\{\left(x_{i}, t_{i}\right)\right\}_{i=1}^{\infty} \subset K \times(0,1]$, and $\lim t_{i}=t_{0}>0$, then $\left\{x_{i}\right\}_{i=1}^{\infty}$ converges in $K$.

First, we derive Proposition 3.1 from Lemmas 3.2 and 3.3.

Proof of 3.1. We make use of 2.1 with the following data: $X=E, Y=\sum_{C} H_{n}$, $Y^{\prime}=\sum_{C} E_{n}$, and $Z$. It is known that $Z$-sets in $E$ are strong $Z$-sets (see [5, Lemma 2.6; 13, Lemma 2.1]). It is also clear that $E \backslash \sum_{C} H_{n}$ and $\sum_{C} E_{n}$ are locally homotopy negligible in $E$ (see, e.g., [17]). Fix a map $\bar{f}: K \rightarrow E$ and open sets $U \subset K$ and $V \subset E$ such that $f=\bar{f} \mid U$ maps $U$ into $V \cap \sum_{C} H_{n}$ and $\bar{f}(x) \notin V$ for all $x \notin U$. Let $\mathscr{V}$ be an open cover of $V$. Pick a map $\omega: V \rightarrow(0,1]$ such that

(1) whenever $y \in V$ and $z \in E$ satisfy ||$y-z|| \mid<2 \omega(y)$ then there exists an element $\mathscr{V}$ containing both $y$ and $z$.

Let $\Phi$ be a homotopy of 3.2. Pick a map $\varepsilon: E \rightarrow[0,1]$ such that

(2) $\varepsilon^{-1}(\{0\})=E \backslash V$,

(3) \|\|$\Phi(y, \varepsilon(y))-y \|<\omega(y)$ for all $y \in V$,

(4) $\left(\frac{1}{\varepsilon(y)}+4\right)^{-1}<\omega(y)$ for all $y \in V$.

Write $\varepsilon(x)=\varepsilon(f(x))$ and $\lambda(x)=\left(\frac{1}{\varepsilon(x)}+4\right)^{-1}$. Pick a homotopy $\varphi$ from 3.3 and define $g: U \rightarrow E$ by the formula

$$
g(x)=\Phi(f(x), \varepsilon(x))+\varphi(x, \lambda(x)) .
$$

Applying (3)-(4) and (v), we get

$$
\text { IIf } f(x)-g(x) \| \mid<\omega(f(x))+\lambda(x)<2 \omega(f(x))
$$

for every $x \in U$. The property (1) of $\omega$ assures that the range of $g$ is $V$ and that $g$ is $\mathscr{V}$-close to $f$. Clearly, $g$ takes values in $\sum_{C} E_{n}$ and, by (iv), $g^{-1}\left(V \cap \sum_{C} H_{n}\right)=L \cap U$.

To finish the proof, it remains to show that $g: U \rightarrow V$ is a closed embedding. First we check that $g$ is one-to-one. If $\frac{1}{n+1}<\varepsilon(x) \leq \frac{1}{n}$ then, by (ii), $\Phi_{p}(f(x), \varepsilon(x))=0$ for all $p \geq n+3$. Since $\frac{1}{n+5}<\lambda(x) \leq \frac{1}{n+4}$ we have, by (vi), $\varphi_{n+6}(x, \lambda(x)) \neq 0$ and $\varphi_{k}(x, \lambda(x))=0$ for $k \neq n+4, n+5, n+6, n+7$. Assume that $g(x)=g\left(x^{\prime}\right)$ and $\varepsilon\left(x^{\prime}\right) \leq \varepsilon(x)$. Letting $\frac{1}{n^{\prime}+1}<\varepsilon\left(x^{\prime}\right) \leq \frac{1}{n^{\prime}}$, we see that $n^{\prime} \geq n$ and, by (vi), $\varphi_{n^{\prime}+6}\left(x^{\prime}, \lambda\left(x^{\prime}\right)\right) \neq 0$. It follows that

$$
\varphi_{n^{\prime}+6}\left(x^{\prime}, \lambda\left(x^{\prime}\right)\right)=g_{n^{\prime}+6}\left(x^{\prime}\right)=g_{n^{\prime}+6}(x)=\varphi_{n^{\prime}+6}(x, \lambda(x)) \neq 0 ;
$$

hence, $n^{\prime}=n$ or $n+1$. Then, for every $p \geq n+4$, we have $\Phi_{p}\left(f\left(x^{\prime}\right), \varepsilon\left(x^{\prime}\right)\right)=$ $\Phi_{p}(f(x), \varepsilon(x))=0$ and consequently $\varphi_{p}(x, \lambda(x))=g_{p}(x)=g_{p}\left(x^{\prime}\right)=$ $\varphi_{p}\left(x^{\prime}, \lambda\left(x^{\prime}\right)\right)$. Since, by $(\mathrm{vi}), \varphi_{p}(x, \lambda(x))=\varphi_{p}\left(x^{\prime}, \lambda\left(x^{\prime}\right)\right)=0$ for all $p \leq n+3$, we conclude that

$$
\varphi(x, \lambda(x))=\varphi\left(x^{\prime}, \lambda\left(x^{\prime}\right)\right) .
$$


The latter yields $x=x^{\prime}$ because $\varphi$ is one-to-one. Now, suppose $\left\{g\left(x_{i}\right)\right\}_{i=1}^{\infty}$ converges to $y=\left(y_{n}\right) \in V$ for some sequence $\left\{x_{i}\right\}_{i=1}^{\infty} \subset U$. Write $\varepsilon_{i}=\varepsilon\left(x_{i}\right)$ and $\lambda_{i}=\lambda\left(x_{i}\right)$. We can assume that $\left\{\varepsilon_{i}\right\}_{i=1}^{\infty}$ converges to $\varepsilon_{0} \in[0,1]$. If $\varepsilon_{0}=0$, then $\lim \lambda_{i}=0$. Using (v), we get $\lim \Phi\left(f\left(x_{i}\right), \varepsilon_{i}\right)=y$. Then, by (iii), $\left\{f\left(x_{i}\right)\right\}_{i=1}^{\infty}$ converges to $y$. By the continuity of $\varepsilon$, we get $\varepsilon(y)=0$ which contradicts (2). Therefore, we can assume that $\varepsilon_{0}>0$. Let

$$
\varepsilon_{0}=s_{0} \frac{1}{n}+\left(1-s_{0}\right) \frac{1}{n+1}
$$

for some $0<s_{0} \leq 1$. We can further assume that

$$
\varepsilon_{i}=s_{i} \frac{1}{n}+\left(1-s_{i}\right) \frac{1}{n \pm 1} \text {. }
$$

Then, we have $\Phi_{n+j}\left(x_{i}, \varepsilon_{i}\right)=0$ for all $i$ and $j \geq 3$; and consequently the sequence $\left\{\varphi_{n+j}\left(x_{i}, \lambda_{i}\right)\right\}_{i=1}^{\infty}=\left\{g_{n+j}\left(x_{i}\right)\right\}_{i=1}^{\infty}$ converges to $y_{n+j}$ for all $j \geq 3$. For $p \neq n+j$, we have, by (vi), $\varphi_{p}\left(x_{i}, \lambda_{i}\right)=0$. It follows that $\left\{\varphi\left(x_{i}, \lambda_{i}\right)\right\}_{i=1}^{\infty}$ converges in $E$. Since $\lim \lambda_{i}=\left(\frac{1}{\varepsilon_{0}}+4\right)^{-1}>0$, by (vii), the sequence $\left\{x_{i}\right\}_{i=1}^{\infty}$ is convergent in $K$. If $\lim x_{i}=x \in K \backslash U$, then $\lim f\left(x_{i}\right)=f(x) \in E \backslash V$ and $\lim \varepsilon\left(x_{i}\right)=\varepsilon(f(x))=0$, contradicting (2). We have shown that $g$ is a closed embedding.

Proof of 3.2. Pick a vector $e_{n} \in H_{n}$ with $\left\|\left|e_{n} \|\right|=1\right.$. Define $\Phi: E \times[0,1] \rightarrow E$ by $\Phi(y, 0)=y$,

$$
\Phi\left(y, \frac{1}{n}\right)=\left(y_{1}, \ldots, y_{n-1}, 0,\left\||| r_{n}(y)\right\| \mid \cdot e_{n+1}, 0,0, \ldots\right)
$$

and

$$
\Phi\left(y, s \frac{1}{n}+(1-s) \frac{1}{n+1}\right)=s \Phi\left(y, \frac{1}{n}\right)+(1-s) \Phi\left(y, \frac{1}{n+1}\right)
$$

for every $n \geq 1,0 \leq s \leq 1$, and $y=\left(y_{n}\right) \in E$. It is clear that $\Phi$ transforms $\sum_{C} H_{n} \times[0,1]$ into $\sum_{C} H_{n}$, is continuous on $E \times(0,1]$, and satisfies (i) and (ii). The continuity of $\Phi$ at the points $(y, 0)$ will follow from the auxiliary estimations.

Given $y=\left(y_{n}\right) \in E$, we have

$$
\begin{aligned}
& \left|\left\|y-\Phi\left(y, \frac{1}{n}\right)|||\leq||| y-s_{n}(y)|\|+||| s_{n}(y)-\Phi\left(y, \frac{1}{n}\right)|| \mid\right.\right. \\
& =\|\| r_{n}(y)\|\|+\|\|||\left|r_{n}(y)\|\| \cdot e_{n+1}\right|\|=2\|\left\|r_{n}(y)\right\| \| .
\end{aligned}
$$

For $t=s \frac{1}{n}+(1-s) \frac{1}{n+1}, 0 \leq s \leq 1$, and $y \in E$, we have

(1) $\mid\|y-\Phi(y, t)\|\|=\| s\left(y-\Phi\left(y, \frac{1}{n}\right)\right)+(1-s)\left(y-\Phi\left(y, \frac{1}{n+1}\right)\right)\|\|$ $\leq s\left|\left\|y-\Phi\left(y, \frac{1}{n}\right)\right\|\|+(1-s)\|\right| y-\Phi\left(y, \frac{1}{n+1}\right) \mid\|\leq 2 s\| r_{n}(y)\|\|+$ $2(1-s)\left|\left\|r_{n+1}(y)\left|\||\leq 2|\| r_{n}(y)\|\|\right.\right.\right.$.

Let $\left\{\left(y(i), t_{i}\right)\right\}_{i=1}^{\infty}$ be a sequence of $E \times(0,1]$ that is convergent to $(y, 0) \in$ $E \times\{0\}$ and let

(2) $t_{i}=s_{i} \frac{1}{n_{i}}+\left(1-s_{i}\right) \frac{1}{n_{i}+1}$ for some $0 \leq s_{i} \leq 1$ and $n_{i} \rightarrow \infty$.

Using (1), we get

(3) $\quad\left\|y-\Phi\left(y(i), t_{i}\right)\right\||\leq\|y-y(i)\||\left|+\left\|\left|y(i)-\Phi\left(y(i), t_{i}\right)\||\leq\|y-y(i)\||+\right.\right.\right.$ $2 \mid\left\|r_{n_{i}}(y(i))\right\| \|$. 
On the other hand, applying (B), we obtain

(4) $\left|\left\|r_{n}(y(i))\left|\||\leq|\| r_{n}(y)\right|\right\|+\right||| r_{n}(y-y(i))\left\||| \leq|| r_{n}(y)|\|+\mid\| y-y(i)\|\|\right.$.

Combining (3) and (4), we get

$$
||\left|y-\Phi\left(y(i), t_{i}\right)\right||| \leq 3|| y-y(i)|||+2||| r_{n_{i}}(y)|| \mid .
$$

The latter inequality together with (C) yields the continuity of $\Phi$ at $(y, 0)$.

Now, let $\left\{\left(y(i), t_{i}\right)\right\}_{i=1}^{\infty} \subset E \times(0,1]$ be such that $\lim \Phi\left(y(i), t_{i}\right)=y \in E$ and $\lim t_{i}=0$. Express $t_{i}$ in the form of (2). We have

$$
\||y-y(i)|\||\leq|\left|s_{n_{i}}(y)-s_{n_{i}}(y(i))\right|||+|| r_{n_{i}}(y)\left|\left\|\left|+\left\|\left|r_{n_{i}}(y(i)) \|\right| .\right.\right.\right.\right.
$$

We see that $s_{n_{i}}(y(i))=s_{n_{i}}\left(\Phi\left(y(i), t_{i}\right)\right)$. Therefore, after using (A), we get

$$
\left\|\left|s_{n_{i}}(y)-s_{n_{i}}(y(i))\|\|=\left\|| | s _ { n _ { i } } ( y - \Phi ( y ( i ) , t _ { i } ) ) \left|\||\leq|\| y-\Phi\left(y(i), t_{i}\right) \| .\right.\right.\right.\right.
$$

This implies

$$
|| y-y(i)\left\||\leq||| y-\Phi\left(y(i), t_{i}\right)\left|\left\|+||\left|r_{n_{i}}(y)\right|||+\right\|\right| r_{n_{i}}(y(i)) \mid\right\| .
$$

Note that the first two terms tend to 0 if $i \rightarrow \infty$. To show (iii), it remains to verify that the last term also tends to 0 . It is clear that it is enough to consider the case where $s_{i} \geq \frac{1}{2}$ for all $i$ and the case where $s_{i}<\frac{1}{2}$ for all $i$. In the first case, we apply (B) to the $\left(n_{i}+1\right)$-coordinate and get

$$
\left\|y-\Phi\left(y(i), t_{i}\right)\left|\|\geq\| y_{n_{i}+1}-s_{i}\left\|\mid r_{n_{i}}(y(i))\right\|\left\|\cdot e_{n_{i}+1}\right\| .\right.\right.
$$

Then, since $\left\|\left|e_{n_{i}+1}\right|\right\|=1$, we estimate

$$
\begin{aligned}
\left\|\mid r_{n_{i}}(y(i))\right\| \| & \leq \frac{1}{s_{i}}\left(\left\||| y-\Phi\left(y(i), t_{i}\right)|\|+\||\left|y_{n_{i}+1} \|\right|\right)\right. \\
& \leq 2\left(\left\||| y-\Phi\left(y(i), t_{i}\right)\left|\left\|\left|+\left\|y_{n_{i}+1}\right\|\right|\right) .\right.\right.\right.
\end{aligned}
$$

Finally, according to (B) and (C), $\lim _{i \rightarrow \infty} r_{n_{i}}(y(i))=0$. In the case where $s_{i}<\frac{1}{2}$, we apply (B) to the $n_{i}$-coordinate and obtain

$$
||\left|y-\Phi\left(y(i), t_{i}\right)\right|\left\|\geq||\left|y_{n_{i}}-\left(1-s_{i}\right) y_{n_{i}}(i)\right|\right\|
$$

and hence

$$
\begin{aligned}
\left\|y_{n_{i}}(i)\right\| \mid & \leq \frac{1}{1-s_{i}}\left(\left\|y-\Phi\left(y(i), t_{i}\right)||\left|+\left\|y_{n_{i}} \mid\right\|\right)\right.\right. \\
& <2\left(\left\||| y-\Phi\left(y(i), t_{i}\right)\left|\left\|+\left|\left\|y_{n_{i}}\right\|\right|\right) .\right.\right.\right.
\end{aligned}
$$

The same argument applied to the $\left(n_{i}+2\right)$-coordinate yields

$$
\left\|\left|y-\Phi\left(y(i), t_{i}\right)\right|\right\||\geq|\left|y_{n_{i}+2}-\left(1-s_{i}\right)\right||| r_{n_{i}+1}(y(i))\left|\left\|\cdot e_{n_{i}+2} \mid\right\| .\right.
$$

As before, we get

$$
\begin{aligned}
\left\|\left|r_{n_{i}+1}(y(i))\right|\right\| & \leq \frac{1}{1-s_{i}}\left(\left\|y-\Phi\left(y(i), t_{i}\right)\left|\left\|\left|+\left\|y_{n_{i}+2} \mid\right\|\right)\right.\right.\right.\right. \\
& \leq 2\left(\left\|y-\Phi\left(y(i), t_{i}\right)\left|\left\|\left|+\| y_{n_{i}+2}\right|||\right) .\right.\right.\right.
\end{aligned}
$$

The latter, in turn, implies

$$
\begin{aligned}
\left\|\mid r_{n_{i}}(y(i))\right\| \| & \leq\left\||| y_{n_{i}}(i)\right\|\|+\| r_{n_{i}+1}(y(i))|\|| \\
& \leq 4\left\|y-\Phi\left(y(i), t_{i}\right)\right\| \mid+2\left(\left\||| y_{n_{i}}\left|\|+\| y_{n_{i}+2} \|\right|\right) .\right.
\end{aligned}
$$

Finally, according to (B) and (C), the last two terms of the above inequality tend to 0 if $i \rightarrow \infty$. 
Note. Condition (iii) is equivalent to the fact that the map $(y, t) \rightarrow(\Phi(y, t), t)$ from $E \times[0,1]$ into $E \times[0,1]$ is closed over $E \times\{0\}$.

Proof of 3.3. By our assumption there exists a closed embedding $\psi_{n}: K \rightarrow E_{n}$ such that

$$
\psi^{-1}\left(H_{n}\right)=L \text { and }\left\|\psi_{n}(x)\right\| \leq \frac{1}{2 n} .
$$

Pick a vector $e_{n} \in H_{n}$ with $\left\|e_{n}\right\|=\frac{1}{2 n}$. Define $\varphi=\left(\varphi_{p}\right)$ as follows:

$$
\varphi_{p}\left(x, \frac{1}{n}\right)= \begin{cases}0 & \text { if } p \neq n, n+2, \\ \psi_{n}(x) & \text { if } p=n, \\ e_{n+2} & \text { if } p=n+2\end{cases}
$$

and

$$
\varphi\left(x, s \frac{1}{n}+(1-s) \frac{1}{n+1}\right)=s \varphi\left(x, \frac{1}{n}\right)+(1-s) \varphi\left(x, \frac{1}{n+1}\right)
$$

for $n \geq 1$ and $0 \leq s \leq 1$. It is clear that $\varphi$ is continuous and satisfies (vi) and (iv). We have

$$
\left\|\left|\varphi ( x , \frac { 1 } { n } ) \left\||| \leq||\left|\psi_{n}(x)\||+|\| e_{n+2} \|\right| \leq \frac{1}{n} .\right.\right.\right.
$$

Consequently, we estimate

$$
\begin{aligned}
\|\varphi(x, t)\| \mid & \leq s\left|\left\|\varphi\left(x, \frac{1}{n}\right)\right\|\right|+(1-s)\left\|\left|\varphi\left(x, \frac{1}{n+1}\right) \|\right|\right. \\
& \leq s \frac{1}{n}+(1-s) \frac{1}{n+1}=t .
\end{aligned}
$$

To show that $\varphi$ is one-to-one, let $\varphi(x, t)=\varphi\left(x^{\prime}, t^{\prime}\right)$ for some $(x, t),\left(x^{\prime}, t^{\prime}\right) \in$ $K \times(0,1]$. If $t=s \frac{1}{n}+(1-s) \frac{1}{n+1}$ with $n \geq 1$ and $0 \leq s<1$ (respectively, $t=1)$, then the last nonvanishing coordinate of $\varphi(x, t)$ is the $(n+3)$ coordinate (respectively, the third coordinate) and it equals $(1-s) e_{n+3}$ (respectively, $\left.e_{3}\right)$. This shows that $t=t^{\prime}$. Clearly, we have $\varphi_{n+1}(x, t)=(1-s) \psi_{n+1}(x)$ (respectively, $\varphi_{1}(x, t)=\psi_{1}(x)$ ). Since $\psi_{n}$ (respectively, $\psi_{1}$ ) is an embedding, we get $x=x^{\prime}$.

Let $\left\{\left(x_{i}, t_{i}\right)\right\}_{i=1}^{\infty}$ be a sequence of $K \times(0,1]$ such that $\left\{\varphi\left(x_{i}, t_{i}\right)\right\}_{i=1}^{\infty}$ converges in $E$ and $\lim t_{i}=t_{0}>0$. Assume that $t_{0}=s_{0} \frac{1}{n}+\left(1-s_{0}\right) \frac{1}{n+1}$ for some $n \geq 1$ and $0<s_{0}<1$ (the case where $t_{0}=\frac{1}{n}, n \geq 1$, can be treated similarly). We may suppose that $t_{i}=s_{i} \frac{1}{n}+\left(1-s_{i}\right) \frac{1}{n+1}$ for all $i$, where $0<s_{i}<1$ and $\lim s_{i}=s_{0}$. Since $\varphi_{n}\left(x_{i}, t_{i}\right)=s_{i} \psi_{n}\left(x_{i}\right),\left\{\psi_{n}\left(x_{i}\right)\right\}_{i=1}^{\infty}$ converges in $E_{n}$. Finally, $\left\{x_{i}\right\}_{i=1}^{\infty}$ converges in $K$ because $\psi_{n}$ is a closed embedding.

In $\S \S 4$ and 5, we will employ the following variation of Proposition 3.1.

Proposition 3.4. Let $\left\{\left(E_{n}, H_{n}\right)\right\}_{n=1}^{\infty}$ be a sequence of pairs of nontrivial normed linear spaces with each $H_{n}$ dense in $E_{n}$ and let $C$ be a normed coordinate space. Fix a pair of spaces $(K, L)$. Assume there are pairwise disjoint infinite subsets $N_{1}, N_{2}, \ldots$ of the set of integers $N$ such that $N_{k} \cap\{1,2, \ldots, k-1\}=\varnothing$ and, writing

$$
C_{k}=\left\{\left(c_{p}\right)_{p \in N_{k}}: \exists_{c \in C} \forall_{p \in N_{k}} \pi_{p}(c)=c_{p}\right\}
$$


and identifying $C_{k}$ with the natural subspace of $C$, each $C_{k}$ contains an element with infinitely many nonzero terms and there exists a bounded closed embedding $\psi_{k}: K \rightarrow \prod_{C_{k}} E_{p}$ with $\psi_{k}^{-1}\left(\prod_{C_{k}} H_{p}\right)=L$ for $k \geq 1$. Then, the pair $\left(\prod_{C} E_{n}, \prod_{C} H_{n}\right)$ is strongly $(K, L)$-universal.

A proof of 3.4 will be omitted. Let us indicate that to get it one has to follow the proof of 3.1 and replace 3.3 by the lemma below.

Lemma 3.5. There exists a one-to-one map

$$
\varphi: K \times(0,1] \rightarrow \prod_{C} E_{n}
$$

satisfying conditions $(\mathrm{v})$ and (vii) of 3.3 together with

(iv') $\varphi^{-1}\left(\prod_{C} H_{n}\right)=L \times(0,1]$,

(vi') given $n \geq 1$ there exists an integer $k_{n}>k_{n-1}\left(k_{1} \geq 1\right)$ such that, if $x \in K$ and $\frac{1}{n+1}<t \leq \frac{1}{n}$ then $\pi_{k_{n}} \varphi(x, t) \neq 0$ while $\pi_{k} \varphi(x, t)=0$ for all $k \in N \backslash\left(N_{n+1} \cup N_{n+2} \cup\left\{k_{n}, k_{n+1}\right\}\right)$.

Proof. Pick $k_{n} \in N_{1}$ with $k_{n}>k_{n-1}\left(k_{1} \geq 1\right)$. By our assumption, there exists a closed embedding $\psi_{n}: K \rightarrow \prod_{C_{n+1}} E_{p}, n \geq 1$, such that

$$
\psi_{n}^{-1}\left(\prod_{C_{n+1}} H_{p}\right)=L \text { and }\left\|\psi_{n}(x)\right\| \leq \frac{1}{n}
$$

for all $x \in K$. Pick a vector $e_{k_{n}} \in H_{k_{n}}$ with $\left\|e_{k_{n}}\right\|=\frac{1}{2 n}$. Define $\varphi=\left(\varphi_{k}\right)$ as

$$
\varphi_{k}\left(x, \frac{1}{n}\right)= \begin{cases}0 & \text { if } k \in N \backslash\left(N_{n+1} \cup\left\{k_{n}\right\}\right), \\ e_{k_{n}} & \text { if } k=k_{n}, \\ \pi_{k} \psi_{n}(x) & \text { if } k \neq k_{n},\end{cases}
$$

and

$$
\varphi\left(x, s \frac{1}{n}+(1-s) \frac{1}{n+1}\right)=s \varphi\left(x, \frac{1}{n}\right)+(1-s) \varphi\left(x, \frac{1}{n+1}\right)
$$

for $n \geq 1$ and $0 \leq s \leq 1$. Conditions $\left(\mathrm{iv}^{\prime}\right)$ and $\left(\mathrm{vi}^{\prime}\right)$ follow easily. To verify (v) and (vii), repeat a reasoning of the proof of 3.3.

The next result is a counterpart of Proposition 3.1 for cartesian products and can be viewed as a relative version of [4, Proposition 2.5]. We need to recall that by the weak product of $X_{i}$ 's with the basepoints $*_{i} \in X_{i}$ we mean

$$
W\left(X_{i}, *_{i}\right)=\left\{\left(x_{i}\right) \in \prod_{i=1}^{\infty} X_{i}: x_{i}=*_{i} \text { for almost all } i\right\}
$$

(endowed with the subspace topology).

Proposition 3.6. Let $X_{i}$ be a noncompact absolute retract and let $Y_{i}$ be a subset of $X_{i}$ such that $X_{i} \backslash Y_{i}$ is locally homotopy negligible in $X_{i}$ for $i=1,2, \ldots$. Fix a pair of spaces $(K, L)$ and assume that for every $i \geq 1$ there exists a closed embedding

$$
h_{i}: K \rightarrow X_{i} \text { with } h_{i}^{-1}\left(Y_{i}\right)=L .
$$

Then, for every choice of basepoints $*_{i} \in Y_{i}$ and every set $Z \subseteq X=\prod_{i=1}^{\infty} X_{i}$ with $Z \cap W\left(X_{i}, *_{i}\right)=W\left(Y_{i}, *_{i}\right)$, the pair $(X, Z)$ is strongly $(K, L)$-universal. Proof. We apply Proposition 2.1 with the following data: $X, Y=W\left(Y_{i}, *_{i}\right)$, $Y^{\prime}=W\left(X_{i}, *_{i}\right)$, and $Z$. It is clear that $X$ is an absolute retract and both $X \backslash Y$ 
and $Y^{\prime}$ are locally homotopy negligible in $X$. By [13, Lemma 2.2] $Z$-sets are strong $Z$-sets in $X$. Let $\bar{f}: K \rightarrow X$ be such that $f=\left(f_{i}\right)=\bar{f} \mid U$ maps $U$ into $V \cap Y$ and $\bar{f}(x) \notin V$ for all $x \notin U$, where $U \subset K$ and $V \subset X$ are open sets. Let $\mathscr{V}$ be an open cover of $V$. We pick a map $\mu: X_{i} \times X_{i} \times[0,1] \rightarrow X_{i}$ such that for all $i \geq 1$

(1) $\mu_{i}(x, y, 0)=x$ and $\mu_{i}(x, y, 1)=y$ for every $x, y \in X_{i}$,

(2) $\mu_{i}\left(Y_{i} \times Y_{i} \times[0,1]\right) \subset Y_{i}$.

To construct $\mu_{i}$, choose any map $\lambda_{i}: X_{i} \times X_{i} \times[0,1] \rightarrow X_{i}$ satisfying (1) and a homotopy $\left(\varphi_{t}^{i}\right): X_{i} \times[0,1] \rightarrow X_{i}$ with $\varphi_{0}^{i}=\operatorname{id}_{X_{i}}$ and $\varphi_{t}^{i}\left(X_{i}\right) \subseteq Y_{i}$ for all $t>0$ and define

$$
\mu_{i}(x, y, t)=\varphi_{t(1-t)}^{i}\left(\lambda_{i}(x, y, t)\right)
$$

To produce $\varphi_{t}^{i}$, use the fact that $X_{i} \backslash Y_{i}$ is locally homotopy negligible in $X_{i}$ and apply [17, Theorem 2.4]. The same property implies that $Y_{i}$ is an absolute retract [17, Theorem 3.1]; moreover, since $X_{i}$ is noncompact, $Y_{i}$ is nontrivial. As a consequence, there exists an embedding $\alpha_{i}:[0,1] \rightarrow Y_{i}$ with

(3) $\alpha_{i}(0)=*_{i}$ for $i \geq 1$.

Fix $t=s \frac{1}{n}+(1-s) \frac{1}{n+1}, n \geq 1$ and $0 \leq s \leq 1$, and let $\Phi(x, t)=\left(y_{i}\right)$, where

(4) $y_{i}=f_{i}(x)$ for $i \leq n$,

(5) $y_{i}=*_{i}$ for $i=n+6$ and $i \geq n+9$,

(6) $y_{n+1}=\mu_{n+1}\left(f_{n+1}(x), *_{n+1}, s\right)$,

(7) $y_{n+2}=\mu_{n+2}\left(*_{n+2}, h_{n+2}(x), s\right)$,

(8) $y_{n+i}=h_{n+i}(x)$ for $i=3$ and 4 ,

(9) $y_{n+5}=\mu_{n+5}\left(h_{n+5}(x), *_{n+5}, s\right)$,

(10) $y_{n+7}=\alpha_{n+7}(s)$ and $y_{n+8}=\alpha_{n+8}(1-s)$.

Letting $\Phi(x, 0)=f(x)$, we easily check that $\Phi: K \times[0,1] \rightarrow X$ is well defined and continuous. Notice that, by $(2)$,

(11) $\Phi(K \times(0,1]) \subset W\left(X_{i}, *_{i}\right)$,

(12) $\Phi^{-1}\left(W\left(Y_{i}, *_{i}\right)\right) \cap(K \times(0,1])=L \times(0,1]$

We claim that $\Phi \mid K \times(0,1]$ is one-to-one. In fact, let $(x, t)$ and $\left(x^{\prime}, t^{\prime}\right)$ be such that $\Phi(x, t)=\Phi\left(x^{\prime}, t^{\prime}\right)$. If $t=s \frac{1}{n}+(1-s) \frac{1}{n+1}, n \geq 1$ and $0 \leq s<1$ (respectively, $t=1$ ), the last $p$ th coordinate of $\Phi(x, t)$, different from ${ }^{*} p$, occurs when $p=n+8$ (respectively, $p=8$ ) and it is equal to $\alpha_{n+8}(1-s)$ (respectively, $\left.\alpha_{8}(1)\right)$. Since $\alpha$ is an embedding, $\Phi(x, t)$ determines $t$ and hence $t=t^{\prime}$. According to (8), $\Phi_{n+3}(x, t)=h_{n+3}(x)$ (respectively, $\Phi_{4}(x, t)=$ $h_{4}(x)$ ). Therefore, $h_{n+3}(x)=h_{n+3}\left(x^{\prime}\right)$ (respectively, $h_{4}(x)=h_{4}\left(x^{\prime}\right)$ ) and consequently we get $x=x^{\prime}$.

Choose a map $\varepsilon: X \rightarrow[0,1]$ such that

(13) $\varepsilon^{-1}(\{0\})=X \backslash V$,

(14) whenever $y \in V$ and $y^{\prime} \in X$ satisfy $d\left(y, y^{\prime}\right)<\varepsilon(y)$ then there is an element of $\mathscr{V}$ containing both $y$ and $y^{\prime}$,

where $d$ is a metric on $X=\prod_{i=1}^{\infty} X_{i}$ chosen so that $d\left(y, y^{\prime}\right)<\frac{1}{n+1}$ if $y$ and $y^{\prime}$ agree on the first $n$ coordinates. By the choice of $d$ and (4), we get

(15) $d(\Phi(x, \varepsilon(f(x))), f(x))<\varepsilon(f(x))$.

To see (15), observe that if $\frac{1}{n+1}<\varepsilon(f(x)) \leq \frac{1}{n}$, then $d(\Phi(x, \varepsilon(f(x))), f(x)) \leq$ 


$$
\begin{aligned}
& \frac{1}{n+1}<\varepsilon(f(x)) \text {. Define } g: U \rightarrow X \text { by } \\
& \qquad g(x)=\Phi(x, \varepsilon(f(x))) .
\end{aligned}
$$

By (14) and (15), $g$ is $\mathscr{V}$-close to $f$ and takes values in $V$. In turn, (5) and (12) imply that $g$ takes values in $W\left(X_{i}, *_{i}\right)$ and satisfies $g^{-1}\left(V \cap W\left(Y_{i}, *_{i}\right)\right)=$ $L \cap U$.

It remains to verify that $g: U \rightarrow V$ is a closed embedding. For some sequence $\left\{x_{k}\right\}_{k=1}^{\infty} \subset U$ let $\lim g\left(x_{k}\right)=y=\left(y_{i}\right) \in V$. We may assume that $\left\{\varepsilon\left(f\left(x_{k}\right)\right)\right\}_{k=1}^{\infty}$ converges to some $\varepsilon_{0} \in[0,1]$. If $\varepsilon_{0}=0$ then, by (15), $\lim f\left(x_{k}\right)=y$, contradicting the fact that $\varepsilon(y)>0$. If $\varepsilon_{0}>0$, then we may assume that that $\varepsilon\left(f\left(x_{k}\right)\right) \in\left(\frac{1}{n+1}, \frac{1}{n-1}\right)$ for some $n$ and all $k$. According to (8), $g_{n+3}\left(x_{k}\right)=h_{n+3}\left(x_{k}\right)$ and consequently $\lim h_{n+3}\left(x_{k}\right)=y_{n+3}$. Since $h_{n+3}$ is a closed embedding, $\left\{x_{k}\right\}_{k=1}^{\infty}$ converges in $K$. If $\lim x_{k}=x \in K \backslash U$, then $\lim f\left(x_{k}\right)=f(x) \notin V$ and, by (13), $\lim \varepsilon\left(f\left(x_{k}\right)\right)=\varepsilon(f(x))=0$, a contradiction.

Note 3.7. Our proof of Proposition 3.6 requires that at least infinitely many of the $X_{n}$ 's are noncompact. Otherwise, it may happen that not all $Z$-sets are strong $Z$-sets in $X$ (see [13]). However, the proof (after minor modifications) still works if one assumes that all the $X_{n}$ 's are nontrivial local compacta.

\section{BORELIAN ABSORBING SETS CAN BE LINEARLY REPRESENTED IN $l^{2}$}

For every countable ordinal $\alpha \geq 0$, by $\mathscr{A}_{\alpha}$ and $\mathscr{M}_{\alpha}$ we denote the additive and multiplicative classes of all absolute Borelian sets of order $\alpha$, respectively. To be more specific, $\mathscr{M}_{0}$ consists of all compacta, $\mathscr{A}_{1}$ consists of all $\sigma$-compact spaces, $\mathscr{M}_{1}=\mathscr{M}$ consists of all completely metrizable spaces, and $\mathscr{M}_{2}$ consists of all absolute $F_{\sigma \delta}$-sets. By $\mathscr{P}_{n}, n \geq 1$, we denote the class of all projective sets of order $n ; \mathscr{P}_{0}=\bigcup_{\alpha} \mathscr{A}_{\alpha}$. A set that does not belong to $\bigcup_{n=1}^{\infty} \mathscr{P}_{n}$ is called nonprojective.

The aim of this section is to find a linear representation of an $\mathscr{A}_{\alpha}$-absorbing set $F_{\alpha}$ (respectively, $\mathscr{M}_{\alpha}$-absorbing set $G_{\alpha}$ ) in $l^{2}$. To perform this, we will make use of $\mathscr{A}_{\alpha}$-absorbing sets $\Lambda_{\alpha}$ and $\mathscr{M}_{\alpha}$-absorbing sets $\Omega_{\alpha}$ constructed in copies $s$ of $l^{2}$ in [4]. By the uniqueness theorem for absorbing sets [4], $F_{\alpha}$ is homeomorphic to $\Lambda_{\alpha}$ and $G_{\alpha}$ is homeomorphic to $\Omega_{\alpha}$. Actually, we show that the pairs $\left(l^{2}, F_{\alpha}\right)$ and $\left(s, \Lambda_{\alpha}\right), \alpha \geq 1$ (respectively, $\left(l^{2}, G_{\alpha}\right)$ and $\left.\left(s, \Omega_{\alpha}\right), \alpha \geq 2\right)$, are homeomorphic. The last is achieved by proving the strong $\left(\mathscr{K}, \mathscr{A}_{\alpha}\right)$ - and $\left(\mathscr{M}, \mathscr{M}_{\alpha}\right)$-universality of suitable pairs. The multiplicative case of order $\alpha=1$ differs from the others and is treated separately in [8]; we include a description of $G_{1}$ in our text in order to formulate the result in full generality.

We briefly recall the definition of $\Lambda_{\alpha}$ and $\Omega_{\alpha}$. Set $\Lambda_{1}=\Sigma \subset R^{\infty}=s$ and $\Omega_{1}=W\left(R^{\infty}, 0\right) \subset\left(R^{\infty}\right)^{\infty}=s$. Inductively, if $\alpha=\beta+1$ let $\Omega_{\alpha}=$ $\Lambda_{\beta}^{\infty} \subset s_{\beta}^{\infty}=s$, where $\Lambda_{\beta}$ is represented in $s_{\beta}$. If $\alpha$ is a limit ordinal let $\Omega_{\alpha}=\prod_{\xi<\alpha} \Lambda_{\xi}^{\infty} \subset \prod_{\xi<\alpha} s_{\xi}^{\infty}=s$, where $\Lambda_{\xi}$ is represented in $s_{\xi}$. Finally, let $\Lambda_{\alpha}=W\left(s_{\alpha} \backslash \Omega_{\alpha}, *\right) \subset s_{\alpha}^{\infty}=s$, where $\Omega_{\alpha}$ is represented in $s_{\alpha}$ and $*$ is an arbitrary basepoint of $s_{\alpha} \backslash \Omega_{\alpha}$. By the Kadec-Anderson theorem [2], the spaces $s$ (in which $\Lambda_{\alpha}$ and $\Omega_{\alpha}$ are represented) are copies of $l^{2}$.

Proposition 4.1 (cf. [4]). For every $\alpha \geq 2$, the pairs $\left(s, \Lambda_{\alpha}\right)$ and $\left(s, \Omega_{\alpha}\right)$ are strongly $\left(\mathscr{M}, \mathscr{A}_{\alpha}\right)$ - and $\left(\mathscr{M}, \mathscr{M}_{\alpha}\right)$-universal, respectively. The pair $\left(s, \Lambda_{1}\right)=$ $\left(R^{\infty}, \Sigma\right)$ is strongly $\left(\mathscr{M}, \mathscr{A}_{1}\right)$-universal. 
Proof. We only present a proof of the multiplicative case. We repeat the argument of [4, Lemma 6.3] and use Proposition 3.6.

To show that $\left(R^{\infty}, \Sigma\right)$ is strongly $\left(\mathscr{M}, \mathscr{A}_{1}\right)$-universal and fix a pair $(K, L) \in\left(\mathscr{M}, \mathscr{A}_{1}\right)$. There exists a closed embedding $h: K \rightarrow R^{\infty}$ with $h^{-1}(\Sigma)=L$. (Take any closed embedding of $K$ into $R^{\infty}$ and compose it with a homeomorphism of $R^{\infty}$ sending $h(L) \cup \Sigma$ onto $\Sigma$; see [2].) Now, by 3.6, $\left(\left(R^{\infty}\right)^{\infty}, W(\Sigma, 0)\right)$ is strongly $\left(\mathscr{M}, \mathscr{A}_{1}\right)$-universal. Since the latter pair is homeomorphic to $\left(R^{\infty}, \Sigma\right)[2$, p. 275$]$, the strong $\left(\mathscr{M}, \mathscr{A}_{1}\right)$-universality of $\left(s, \Lambda_{1}\right)$ follows.

We assume that $\alpha \geq 2$ and $\alpha=\beta+1$ (the case of a limit ordinal is analogous). Given $(K, L) \in\left(\mathscr{M}, \mathscr{M}_{\alpha}\right)$ there exists $L_{i} \subset K, L_{i} \in \mathscr{A}_{\beta} \quad(i \geq 1)$, such that $L=\bigcap_{i=1}^{\infty} L_{i}$. By the inductive assumption, we find a closed embedding $h_{i}: K \rightarrow s_{\beta}$ with $h^{-1}\left(\Lambda_{\beta}\right)=L_{i}$. Writing $h=\left(h_{i}\right)$, we see that $h$ is a closed embedding of $K$ into $s_{\beta}^{\infty}=s$ with $h^{-1}\left(\Omega_{\alpha}\right)=\bigcap_{i=1}^{\infty} L_{i}=L$. Finally, Proposition 3.6 yields the strong $\left(\mathscr{M}, \mathscr{M}_{\alpha}\right)$-universality of $\left(s_{\beta}^{\infty}, \Lambda_{\beta}^{\infty}\right)=\left(s, \Omega_{\alpha}\right)$.

Theorem 4.2. For every $\alpha \geq 1$, there exists a linear subspace $F_{\alpha}$ of $l^{2}$ which is an $\mathscr{A}_{\alpha}$-absorbing set and such that the pair $\left(l^{2}, F_{\alpha}\right)$ is strongly $\left(\mathscr{M}, \mathscr{A}_{\alpha}\right)$ universal. In particular, $\left(l^{2}, F_{\alpha}\right)$ and $\left(s, \Lambda_{\alpha}\right)$ are homeomorphic.

Proof. Construction of $F_{\alpha}$. Let $(A, B)$ be a copy of $\left(s, \Lambda_{\alpha}\right)$. Consider a closed embedding $h$ of $A$ onto a linearly independent subset of the unit sphere in $l^{2}$ satisfying the following condition:

(*) for every $a \in A$ and every closed subset $F \subset A$ with $a \notin F$ there exists a continuous linear functional $x^{*}: l^{2} \rightarrow R$ such that $x^{*}(h(F)) \subseteq\{0\}$ while $x^{*}(h(a)) \neq 0$.

Condition $(*)$ is taken from [3] where it was checked that the embedding described by Bessaga and Petczyński [2, p. 193] fulfils (*). Denote by $H$ the linear span of $h(B)$ in $l^{2}$ and by $\bar{H}$ the closure of $H$. Since $B$ is dense in $A, \bar{H}$ contains $h(A)$ as a (closed) subset. Write $\left(E_{n}, H_{n}\right)=(\bar{H}, H)$ and set

$$
E=\prod_{l^{2}} E_{n} \text { and } F_{\alpha}=\sum_{l^{2}} H_{n} .
$$

Clearly, $E$ is isomorphic to $l^{2}$ and $F_{\alpha}$ is dense in $E$.

According to 4.1 and the fact that $\Lambda_{\alpha}$ is an $\mathscr{A}_{\alpha}$-absorbing set [4], it suffices to prove that the pair $\left(E, F_{\alpha}\right)$ fulfils the requirements (i)-(iv) of 2.2 for the class $\mathscr{L}=\mathscr{A}_{\alpha}$. Condition (i) is a consequence of the fact that $F_{\alpha}$ is linear and dense in $E$ (see, e.g., [17, Remark 2.9]). Since each set $A_{k}=$ $\left\{\left(y_{n}\right) \in F_{\alpha}: y_{i}=0\right.$ for $\left.i \geq k+1\right\}$ is a $Z$-set in $F_{\alpha}$ and $F_{\alpha}=\bigcup_{k=1}^{\infty} A_{k}, F_{\alpha}$ is a $Z_{\sigma}$-space. Condition (iv) follows directly from 3.1 and 4.1 because $A$ is closed in $E_{n}$. The remaining condition (iii) can be concluded from (ii) and the lemma below.

Lemma 4.3. The space $H$ is in $\mathscr{A}_{\alpha}$.

Proof. We shall make use of the cross-section argument due to Klee [2, p. 271]. The $n$-fold product $B^{n}$ admits a $\sigma$-closed cross-section, i.e., there exists a subset $F$ of $B^{n}$ that is a countable union of closed sets $F_{k}$ such that

(1) if $\left(b_{1}, b_{2}, \ldots, b_{n}\right) \in F$ then $b_{i} \neq b_{j}$ for $i \neq j$,

(2) whenever $\left\{y_{i}\right\}_{i=1}^{n}$ are $n$ distinct points of $B$ then there exists exactly one permutation of $y_{1}, y_{2}, \ldots, y_{n}$ that belongs to $F$. 
By (1) and (2) the linear combination map $\chi$ given by

$$
\left(\left(b_{1}, b_{2}, \ldots, b_{n}\right),\left(\lambda_{1}, \lambda_{2}, \ldots, \lambda_{n}\right)\right) \rightarrow \lambda_{1} h\left(b_{1}\right)+\lambda_{2} h\left(b_{2}\right)+\cdots+\lambda_{n} h\left(b_{n}\right)
$$

transforms in a one-to-one way the product $F_{k} \times D_{k}^{p}$ onto $N_{k}^{p} \subset H$, where

$$
D_{k}^{p}=\left\{\left(\lambda_{1}, \lambda_{2}, \ldots, \lambda_{n}\right): \frac{1}{p} \leq\left|\lambda_{i}\right| \leq p \text { for all } i\right\}
$$

and $p=1,2, \ldots$ Employing, as in [3, Lemma 3.3], the condition $(*)$ one shows that $\chi \mid F_{k} \times D_{k}^{p}$ is a homeomorphism. It shows that each $N_{k}^{p}$, and hence $H^{n}=\bigcup_{k, p=1}^{\infty} N_{k}^{p}$, belongs to $\mathscr{A}_{\alpha}$. Since $H=\bigcup_{n=1}^{\infty} H^{n}$, we get $H \in \mathscr{A}_{\alpha}$.

Theorem 4.4. For every $\alpha \geq 1$, there exists a linear subspace $G_{\alpha}$ of $l^{2}$ which is an $\mathscr{M}_{\alpha}$-absorbing set and such that the pair $\left(l^{2}, G_{\alpha}\right)$ is strongly $\left(\mathscr{M}, \mathscr{M}_{\alpha}\right)$ universal. In particular, $\left(l^{2}, G_{\alpha}\right)$ and $\left(s, \Omega_{\alpha}\right)$ are homeomorphic for $\alpha \geq 2$.

Proof. The case for $\alpha=1$ differs from the others. In [8] it was shown that the space

$$
G_{1}=\left\{\left(x_{n}\right) \in l^{2} ; \sum_{n=1}^{\infty}\left|x_{n}\right|<\infty \text { and } \sum_{n=1}^{\infty} x_{n}=0\right\}
$$

is an $\mathscr{M}$-absorbing set and, moreover, the pair $\left(l^{2}, G_{1}\right)$ is strongly $(\mathscr{M}, \mathscr{M})$ universal.

Construction of $G_{\alpha}$. Let $\alpha \geq 2$. If $\alpha$ is a limit ordinal, then choose an increasing sequence of ordinals $\left\{\beta_{n}\right\}_{n=1}^{\infty}$ convergent to $\alpha$; otherwise, $\alpha=\beta+1$ and let $\beta_{n}=\beta$. Pick, by 4.2 , a pair $\left(E_{n}, F_{n}\right)=\left(l^{2}, F_{\beta_{n}}\right)$ which is $\left(\mathscr{M}, \mathscr{A}_{\beta_{n}}\right)$ universal. Set

$$
E=\prod_{l^{2}} E_{n} \text { and } G_{\alpha}=\prod_{l^{2}} F_{n} .
$$

The space $E$ is isomorphic to $l^{2}$ and $G_{\alpha}$ is its linear dense subspace.

Using 4.1 and the fact that $\Omega_{\alpha}$ is an $\mathscr{M}_{\alpha}$-absorbing set [4], it is enough to verify conditions (i)-(iv) of 2.2 for the pair $\left(E, G_{\alpha}\right)$ and $\mathscr{L}=\mathscr{M}_{\alpha}$. Condition (i) follows as in the proof of 4.2. A simple argument shows that $G_{\alpha} \in \mathscr{M}_{\alpha}$. Since $F_{1}$ is a $Z_{\sigma}$-space, $G_{\alpha}$ is also a $Z_{\alpha}$-space. It remains to verify that $\left(E, G_{\alpha}\right)$ is strongly $\left(\mathscr{M}, \mathscr{M}_{\alpha}\right)$-universal. We will apply 3.4 . Let $N_{1}, N_{2}, \ldots$ be any decomposition of the set of integers $N$ into pairwise disjoint infinite sets. Then, the space $C_{k}$ defined in 3.4 is $l^{2}\left(N_{k}\right)$, the space of all square summable sequences indexed by the integers of $N_{k}$. As a result $\left(\prod_{C_{k}} E_{p}, \prod_{C_{k}} H_{p}\right)=$ $\left(\prod_{l^{2}\left(N_{k}\right)} E_{p}, \prod_{l^{2}\left(N_{k}\right)} F_{p}\right)$. By the choice of $\beta_{n}$, it is clear that the following lemma will finish the proof of 4.4 .

Lemma 4.5. For every $(K, L) \in\left(\mathscr{M}, \mathscr{M}_{\alpha}\right)$ there exists a bounded closed embedding $\psi: K \rightarrow E$ with $\psi^{-1}\left(G_{\alpha}\right)=L$.

Proof. Let $L=\bigcap_{k=1}^{\infty} L_{k}$, where $L_{k} \subset K, L_{k+1} \subset L_{k}$, and $L_{k} \in \mathscr{A}_{\beta_{n_{k}}}$ for some $n_{k}$ with $n_{k+1}>n_{k}$. Write $\beta(k)=\beta_{n_{k}}$. Since $\left(E_{n_{k}}, F_{n_{k}}\right)$ is strongly $\left(\mathscr{K}, \mathscr{A}_{\beta(k)}\right)$-universal, there exists a closed embedding $\psi_{n_{k}}: K \rightarrow E_{n_{k}}$ such that

(1) $\left\|\psi_{n_{k}}(x)\right\| \leq\left(\frac{1}{2}\right)^{n_{k}}$ for all $x \in K$,

(2) $\psi_{n_{k}}^{-1}\left(F_{n_{k}}\right)=L_{k}$. 
Write $\psi_{k} \equiv 0$ for all $n \neq n_{k}(k \geq 1)$ and set $\psi=\left(\psi_{n}\right)$. By $(1), \psi$ is continuous and bounded. It is easy to see that $\psi: K \rightarrow E$ is a closed embedding with $\psi^{-1}\left(G_{\alpha}\right)=\bigcap_{k=1}^{\infty} L_{k}=L$.

Remark 4.6. As pointed out in [8], the pair $\left(s, \Omega_{1}\right)=\left(\left(R^{\infty}\right)^{\infty}, W\left(R^{\infty}, 0\right)\right)$ is not strongly $(\mathscr{M}, \mathscr{M})$-universal. (If it were, then by $2.2,\left(s, \Omega_{1}\right)$ and $\left(l^{2}, G_{1}\right)$ would be homeomorphic; consequently, $G_{1}$ would be $\sigma$-closed in $l^{2}$, which contradicts a result of [15].)

Remark 4.7. The spaces $\Lambda_{\alpha}$ and $\Omega_{\alpha}$ can be realized as linear subspaces in other normed coordinate products $\prod_{C} E_{n}$. The only restriction is the condition $(*)$.

Remark 4.8. The result of 4.1 can be readily generalized to the triple case. Representing $\Lambda_{\alpha}(\alpha \geq 1)$ and $\Omega_{\alpha}(\alpha \geq 2)$ in $R^{\infty}$, we could consider the triples

$$
\left(\bar{R}^{\infty}, R^{\infty}, \Lambda_{\alpha}\right) \text { and }\left(\bar{R}^{\infty}, R^{\infty}, \Omega_{\alpha}\right),
$$

where $\bar{R}=[-\infty,+\infty]$. These triples are strongly $\left(\mathscr{M}_{0}, \mathscr{M}, \mathscr{A}_{\alpha}\right)$ - and $\left(\mathscr{M}_{0}, \mathscr{M}, \mathscr{M}_{\alpha}\right)$-universal, respectively (with an obvious meaning of the triple strong universality). $G_{\alpha}$.

In $\S 6$ we shall need the following fact concerning the complements of $F_{\alpha}$ and

Corollary 4.9. The space $l^{2} \backslash F_{\alpha}$ (respectively, $l^{2} \backslash G_{\alpha}$ ) has the following properties:

(i) $l^{2} \backslash F_{\alpha}$ (respectively, $l^{2} \backslash G_{\alpha}$ ) is a Baire space,

(ii) $l^{2} \backslash F_{\alpha} \in \mathscr{M}_{\alpha} \backslash \mathscr{A}_{\alpha}$ (respectively, $l^{2} \backslash G_{\alpha} \in \mathscr{A}_{\alpha} \backslash \mathscr{M}_{\alpha}$ ),

(iii) $l^{2} \backslash F_{\alpha}$ (respectively, $l^{2} \backslash G_{\alpha}$ ) is homogeneous,

(iv) for every (closed) ball $B \subset l^{2}, B \backslash F_{\alpha}$ (respectively, $B \backslash G_{\alpha}$ ) is an absolute retract.

Proof. We will only deal with the $F_{\alpha}$-case, the $G_{\alpha}$-case is analogous. Conditions (i) and (ii) follow from the fact that $F_{\alpha}$ is of the first category and that $F_{\alpha} \in$ $\mathscr{A}_{\alpha} \backslash \mathscr{M}_{\alpha}$. To show (iii), we produce a homeomorphism of $l^{2}$ that preserves $F_{\alpha}$ and carries $y \in l^{2} \backslash F_{\alpha}$ onto $y^{\prime} \in l^{2} \backslash F_{\alpha}$. Let $h$ be any homeomorphism of $l^{2}$ with $h(y)=y^{\prime}$, e.g., $h$ is the translation. Then, the pairs $\left(l^{2}, h\left(F_{\alpha}\right)\right)$ and $\left(l^{2}, F_{\alpha}\right)$ are strongly $\left(\mathscr{M}, \mathscr{A}_{\alpha}\right)$-universal. The proof of [7, Theorem 2.1$]$ can be easily modified to achieve a homeomorphism $k$ of $l^{2}$ that carries $h\left(F_{\alpha}\right)$ onto $F_{\alpha}$ and preserves $\left\{y^{\prime}\right\}$ (set $X_{0}=Y_{0}=\left\{y^{\prime}\right\} \subset X_{1} \cap Y_{1}$ ). We see that $k \circ h$ preserves $F_{\alpha}$ and sends $y$ onto $y^{\prime}$. Condition (iv) is a consequence of the fact that $B \cap F_{\alpha}$ is locally homotopy negligible in $B$ and [17, Theorem 3.1]. Assume $0 \in$ int $B$ and pick $y_{0} \in B \backslash F_{\alpha}$. Since the homotopy $f_{t}(y)=(1-t) y+t y_{0}$ $(0 \leq t \leq 1)$ takes its values in $B \backslash F_{\alpha}$ for $t>0$ and $f_{0}=\mathrm{id}$, the local homotopy negligibility of $B \cap F_{\alpha}$ in $B$ follows.

\section{ApPlication to $F_{\sigma \delta}$-SPACES}

In this section we identify various absolute $F_{\sigma \delta}$-sets carrying product structures to be homeomorphic to $\Omega_{2}=\Sigma^{\infty}$. The spaces we deal with will be considered with both normed and cartesian product topologies. We start with a direct application of Proposition 3.4 to coordinate products of normed $F_{\sigma \delta^{-}}$ spaces. A counterpart of 5.1 for cartesian products was previously obtained in [13]. 
Theorem 5.1. Let $\prod_{C} H_{n}$ be a normed coordinate product of absolute $F_{\sigma \delta}$-spaces $H_{n}$ in the sense of a Banach space $C$. Assume that infinitely many of the $H_{n}$ 's are $Z_{\sigma}$-spaces. Then $\prod_{C} H_{n}$ is homeomorphic to $\Omega_{2}$. Moreover, writing $E_{n}$ for the linear completion of $H_{n}$, the pairs $\left(\prod_{C} E_{n}, \prod_{C} H_{n}\right)$ and $\left(s, \Omega_{2}\right)$ are homeomorphic.

We shall make use of the two lemmas below. A proof of the first one is implicitly contained in [12, Lemma 5.4] and therefore it will be omitted.

Lemma 5.2. Let $X \in \mathscr{M}$ be an absolute retract and $Y \subset X$ be a $Z_{\sigma}$-space such that $X \backslash Y$ is locally homotopy negligible in $X$. Then, for every $L \in \mathscr{A}_{1}$ of the Hilbert cube $I^{\infty}$, there exists a map $\varphi: I^{\infty} \rightarrow X$ with $\varphi^{-1}(Y)=L$.

Lemma 5.3. Let $\left(H_{n},\|\cdot\|_{H_{n}}\right)$ be a normed linear space that is noncompactly embedded into a Banach space $\left(E_{n},\|\cdot\|_{E_{n}}\right)$, i.e., $H_{n} \subseteq E_{n},\|\cdot\|_{E_{n}} \leq\|\cdot\|_{H_{n}}$, and the $E_{n}$-closures of $H_{n}$-balls are noncompact. Then, for every coordinate Banach space $C$ and every $K \in \mathscr{M}$, there exists a bounded closed embedding $\psi: K \rightarrow \prod_{C} E_{n}$ such that $\psi(K) \subset \prod_{C} H_{n}$.

Proof. Since $C$ is complete, there is $c=\left(c_{n}\right) \in C$ with all the $c_{n}$ 's strictly positive. It is enough to construct a closed embedding $\psi=\left(\psi_{n}\right): K \rightarrow \prod_{C} E_{n}$ with $\psi_{n}(x) \in H_{n}$ and $\left\|\psi_{n}(x)\right\|_{H_{n}} \leq c_{n}$ for all $x \in K$ and $n \geq 1$. Fix a metric $d$ on the Hilbert cube $I^{\infty}=[0,1]^{\infty}$. Embed $K$ into $I^{\infty}$ and write $I^{\infty} \backslash K=$ $\bigcup_{n=1}^{\infty} F_{n}$, where each $F_{n}$ is a closed subset of $I^{\infty}$. Define $d_{n}(q)=\operatorname{dist}_{d}\left(q, F_{n}\right)$, $q \in I^{\infty}$. We claim that there exists a closed embedding $\alpha_{n}:[0, \infty) \rightarrow E_{n}$ such that $\alpha_{n}(t) \in H_{n}$ and $\left\|\alpha_{n}(t)\right\|_{H_{n}} \leq 1$ for all $t \geq 0$ and $n \geq 1$. This follows from the noncompactness of the $E_{n}$-closure of the $H_{n}$-unit ball and Klee's result [14] that every noncompact closed convex subset $F$ of $E_{n}$ contains a copy of $[0, \infty)$. (The piecewise linear embedding $\alpha$ constructed by Klee can be improved to get the nodes of $\alpha$ contained in any dense linear subset of $F$.) Pick a vector $e_{2 n} \in H_{2 n}$ with $\left\|e_{2 n}\right\|_{H_{2 n}}=1$. Define $\psi=\left(\psi_{n}\right)$ by

$$
\psi_{2 n}(q)=c_{2 n} q_{n} e_{2 n} \quad \text { and } \quad \psi_{2 n-1}(q)=c_{2 n-1} \alpha_{2 n-1}\left(\left(d_{n}(q)\right)^{-1}\right)
$$

for $q=\left(q_{n}\right) \in K$. It is clear that $\psi: K \rightarrow \prod_{C} E_{n}$ is one-to-one and $\left\|\psi_{n}(q)\right\|_{H_{n}} \leq$ $c_{n}, n \geq 1, q \in K$. By [16, Lemma 1.4], $\psi$ is continuous. If $\{\psi(q(i))\}_{i=1}^{\infty}$ is convergent in $\prod_{C} E_{n}$, then there exists $q_{0} \in I^{\infty}$ with $\lim q(i)=q_{0}$. Assume that $q_{0} \in F_{k}$ for some $k$. Then $\lim _{i \rightarrow \infty} d_{k}(q(i))=0$, contradicting the fact that $\left\{\left(d_{k}(q(i))\right)^{-1}\right\}_{i=1}^{\infty}$ converges. The latter is a consequence of the facts that the sequence $\left\{\alpha_{2 k-1}\left(\left(d_{k}(q(i))\right)^{-1}\right)\right\}_{i=1}^{\infty}$ is convergent in $E_{k}$ and that $\alpha$ is a closed embedding.

Proof of 5.1. We show that the pair $(E, H)=\left(\prod_{C} E_{n}, \prod_{C} H_{n}\right)$ satisfies (i)(iv) of 2.2 with $\mathscr{L}=\mathscr{M}_{2}$. By the Kadec-Anderson theorem [2], $E$ is a copy of $l^{2}$. A standard argument yields (i)-(iii). The strong $\left(\mathscr{M}, \mathscr{M}_{2}\right)$-universality of $(E, H)$ will be derived from Proposition 3.4. Fix a pair $(K, L) \in\left(\mathscr{M}, \mathscr{M}_{2}\right)$. Find pairwise disjoint infinite subsets $N_{1}, N_{2}, \ldots$ of $N$ so that $H_{p}$ is a $Z_{\sigma^{-}}$ space for every $p \in \bigcup_{k=1}^{\infty} N_{k}$. Let $C_{k}$ be the subspace of $C$ that corresponds to $N_{k}$ (see 3.4). Write $E^{k}=\prod_{C_{k}} E_{p}$ and $H^{k}=\prod_{C_{k}} H_{p}$. To fulfil the hypothesis of 3.4 we have to produce a bounded closed embedding $\psi_{k}: K \rightarrow E^{k}$ with $\psi_{k}^{-1}\left(H^{k}\right)=L$. To this end we split $C_{k}=C_{k}^{1} \oplus C_{k}^{2}$ into two coordinate spaces and find a bounded closed embedding $\psi_{k}^{1}: K \rightarrow \prod_{C_{k}^{1}} E_{p}$ with $\psi_{k}^{1}(K) \subset \prod_{C_{k}^{1}} H_{p}$ 
and a bounded map $\psi_{k}^{2}: K \rightarrow \prod_{C_{k}^{2}} E_{p}$ with $\left(\psi_{k}^{2}\right)^{-1}\left(\prod_{C_{k}^{2}} H_{p}\right)=L$. Finally, letting $\psi_{k}=\left(\psi_{k}^{1}, \psi_{k}^{2}\right)$ we get a required embedding (we identify $\prod_{C_{k}} E_{p}$ with $\left.\prod_{C_{k}^{1}} E_{p} \oplus \prod_{C_{k}^{1}} E_{p}\right)$.

To find $\psi_{k}^{1}$ we apply Lemma 5.3. In this case $H_{p}$ is a genuine subspace of an infinite-dimensional Banach space $E_{p}$; hence the balls in $E_{p}$ are noncompact. Let $c=\left(c_{p}\right) \in C_{k}^{2}$ be such that all $c_{p}$ are strictly positive. Embed $K$ into $I^{\infty}$ and represent $L=\bigcap_{p} L_{p}$ so that each $L_{p}$ is $\sigma$-compact and $\left\{L_{p}\right\}$ is descending. Use Lemma 5.2 with $X=B\left(c_{p}\right)$, the closed ball in $E_{p}$ centered at 0 with radius $c_{p}, Y=B\left(c_{p}\right) \cap H_{p}$, and $L=L_{p}$. We get maps $\varphi_{p}: I^{\infty} \rightarrow B\left(c_{p}\right)$ with $\varphi_{p}^{-1}(Y)=L_{p}$. Finally, we set $\psi_{k}^{2}(x)=\left(\varphi_{p}(x)\right), x \in K$. The continuity of $\psi_{k}^{2}$ follows from [16, Lemma 4.1]. To verify the hypothesis of 5.2, notice that $Y$ is convex and dense in $X$. Let $H_{p}=\bigcup_{m=1}^{\infty} A_{m}$, where each $A_{m}$ is a $Z$-set in $H_{p}$. Then $\operatorname{int}\left(B\left(c_{p}\right)\right) \cap A_{m}$ is a $Z$-set in $\operatorname{int}\left(B\left(c_{p}\right)\right) \cap H_{p}$. Since $\operatorname{int}\left(B\left(c_{p}\right)\right) \cap H_{p}$ is convex and dense in $B\left(c_{p}\right) \cap H_{p}, B\left(c_{p}\right) \cap A_{m}$ is a $Z$-set in $B\left(c_{p}\right) \cap H_{p}$. It shows that $Y$ is a $Z_{\sigma}$-space.

\section{A direct consequence of 5.1 is}

Note 5.4. The simplest pre-Hilbert space representation of $\Omega_{2}$ is the space $\prod_{l^{2}} l_{f}^{2}$, where $l_{f}^{2}=\left\{\left(x_{i}\right) \in l^{2}: x_{i}=0\right.$ for almost all $\left.i\right\}$. Moreover, the pairs $\left(\prod_{l^{2}} l^{2}, \prod_{l^{2}} l_{f}^{2}\right)$ and $\left(s, \Omega_{2}\right)$ are homeomorphic.

Consider the set $\prod_{C} H_{n}=H$ as a subspace of the cartesian product $\prod_{n=1}^{\infty} E_{n}$ $=E$. By the Kadec-Anderson theorem [2], $E$ is a copy of $l^{2}$. Easily, $E \backslash H$ is locally homotopy negligible in $E$. We claim that $C$ is an $F_{\sigma \delta}$-subset of $R^{\infty}$. This is a consequence of the equality

$$
C=\left\{\left(x_{n}\right) \in R^{\infty}: \forall_{\varepsilon>0} \exists_{k} \forall_{m>k}\left\|\sum_{i=k}^{m} x_{i} u_{i}\right\|_{C} \leq \varepsilon\right\}
$$

( $u_{i}$ is the $i$ th unit vector). Consider the map $f(x)=\left(\left\|x_{n}\right\|\right), x=\left(x_{n}\right) \in E$, and notice that $f^{-1}(C)=\prod_{C} E_{n}$. This shows that $\prod_{C} E_{n} \in \mathscr{M}_{2}$. Since $\prod_{C} H_{n}=\prod_{C} E_{n} \cap \prod_{n=1}^{\infty} H_{n}, H$ is an absolute $F_{\sigma \delta}$-set. Repeating (with obvious changes) the remaining part of the proof of 5.1, we get the following generalization of a result [13].

Theorem 5.5. Let $\left\{H_{n}\right\}_{n=1}^{\infty}$ be a sequence of normed linear spaces such that each $H_{n}$ is an absolute $F_{\sigma \delta}$-set and infinitely many of the $H_{n}$ 's are $Z_{\sigma}$-spaces. Then, for every coordinate Banach space $C$, the space $\prod_{C} H_{n}$ considered in the product topology, is homeomorphic to $\Omega_{2}$. Moreover, if $E_{n}$ is the linear completion of $H_{n}$ then the pairs $\left(\prod_{n=1}^{\infty} E_{n}, \prod_{C} H_{n}\right)$ and $\left(s, \Omega_{2}\right)$ are homeomorphic.

Remark 5.6. The hypothesis that infinitely many of the $H_{n}$ 's are $Z_{\sigma}$-spaces is essential. Consider the coordinate space $c_{0}=\left\{\left(x_{i}\right) \in R^{\infty}: \lim x_{i}=0\right\}$ with the $\|\cdot\|_{\infty}$-norm. Note that $c_{0} \subset \bigcup_{k=1}^{\infty} B_{\infty}(k)$, where $B_{\infty}(k)=\left\{x \in R^{\infty}\right.$ : $\left.\|x\|_{\infty} \leq k\right\}$. This shows that $c_{0}$ is contained in a $\sigma$-compact subset of $R^{\infty}$. On the other hand $\Omega_{2}$ contains a copy of $R^{\infty}$ closed in $s$. This shows that $\left(R^{\infty}, \Pi_{c_{0}} R\right)$ and $\left(s, \Omega_{2}\right)$ are not homeomorphic, contrary to the expectation expressed in [13]. Proposition 3.6 and Lemma 5.2 yield the strong $\left(\mathscr{M}_{0}, \mathscr{M}_{2}\right)$ universality of the pair $\left(R^{\infty}, c_{0}\right)$. 
For the $c_{0}$-products we have the following generalization of [13, Theorem 4.2].

Theorem 5.7. Let, for $n \geq 1, X_{n}$ be a subset of a Banach space $\left(E_{n},\|\cdot\|_{n}\right)$ with $0 \in X_{n}$ so that $\inf _{n \geq 1} \operatorname{diam}\left(X_{n}\right)=\alpha>0$. Assume that each $X_{n}$ is an absolute retract that is an absolute $F_{\sigma \delta}$-set. Then the space

$$
X=\prod_{c_{0}} X_{n}=\left\{\left(x_{n}\right) \in \prod_{n=1}^{\infty} X_{n} ;\left\|x_{n}\right\|_{n} \rightarrow 0\right\}
$$

(endowed with the product topology) is homeomorphic to $\Omega_{2}$.

Proof. We will show that $X$ is an $\mathscr{M}_{2}$-absorbing set in some copy of $l^{2}$, i.e., we will verify conditions (i)-(iii) of 2.2 and the strong $\mathscr{M}_{2}$-universality of $X$. Then, the uniqueness theorem for absorbing sets [4] yields our assertion. Since $\prod_{n=1}^{\infty} X_{n}$ is an absolute retract and $\prod_{n=1}^{\infty} X_{n} \backslash X$ is locally homotopy negligible, $X$ is also an absolute retract [17, Theorem 3.1]. Decompose the set of integers $N$ into pairwise disjoint infinite sets $N_{1}, N_{2}, \ldots$. Write $X^{i}=\prod_{p \in N_{i}} X_{p}$ and $Y^{i}=\left\{\left(x_{p}\right) \in X^{i}:\left\|x_{p}\right\|_{p} \rightarrow 0\right\}$ and let $\Psi: \prod_{n=1}^{\infty} X_{n} \rightarrow \prod_{i=1}^{\infty} X^{i}$ be the natural isomorphism. We have

(1) $\Psi(X) \supset W\left(Y^{i}, 0\right)$.

Note that each $Y^{i}$ is noncompact. (If it were compact then, because $Y^{i}$ is dense in $X^{i}$, we would get $X^{i}=Y^{i}$, contradicting the fact that $\alpha>0$.) Now, each $Y^{i}$ has a completion $Z^{i}$ with $Z^{i} \backslash Y^{i}$ locally homotopy negligible in $Z^{i}$ [17, Proposition 4.1]. Since $Y^{i}$ is noncompact, we can assume that $Z^{i}$ is also noncompact (if it were compact take $Z^{i} \backslash\{*\}$, where $* \in Z^{i} \backslash Y^{i}$ ). Then the product $s=\prod_{i=1}^{\infty} Z^{i}$ is a copy of $l^{2}[18$, Theorem 5.1] with $s \backslash X$ locally homotopy negligible in $s$; this shows (i). An argument preceding Theorem 5.5 applies to show that $X$ is an absolute $F_{\sigma \delta}$-set. Writing $A_{m}=\left\{\left(x_{n}\right) \in X\right.$ : $\left\|x_{j}\right\|_{j} \leq \frac{\alpha}{2}$ for all $\left.j \geq m+1\right\}$, we see that $\bigcup_{m=1}^{\infty} A_{m}=X$ and that each $A_{m}$ is a $Z$-set in $X$. In proving the strong $\mathscr{M}_{2}$-universality of $X$ we employ 3.6 with $Y_{i}=Y^{i}, K=L \in \mathscr{M}_{2}$, and $Z=\Psi(X)$. To produce a closed embedding of $L$ into $Y^{i}$, we may assume that $Y^{i}=X$.

Write $B^{i}(\varepsilon)=\left\{\left(x_{p}\right) \in X^{i}:\left\|x_{p}\right\|_{p} \leq \varepsilon\right.$ for all $\left.p \in N_{i}\right\}$ and notice that

(2) $\prod_{i=1}^{\infty} Y^{i} \cap B^{i}\left(2^{-i}\right)$ is a closed subset of $\Psi(X)$.

By (2) and the fact that a countable product of $Z_{\sigma}$-spaces that are absolute retracts contains a closed copy of $\Omega_{2}$ [13, Corollary 2.5], it suffices to check that each $Y^{i} \cap B^{i}\left(2^{-i}\right)$ contains a closed $Z_{\sigma}$-space that is an absolute retract. Assuming $\alpha \geq 2^{-i}$, we choose in each $X_{p}$ an arc $T_{p}$ joining 0 with some $x_{p}$ with $\left\|x_{p}\right\|_{p}=2^{-i}$. Write $T^{i}=Y^{i} \cap \prod_{p \in N_{i}} T_{p}$. Then $T^{i}$ is a closed subset of $Y^{i}$. The argument showing that $X$ is a $Z_{\sigma}$-space applies also to verify that $T^{i}$ is a $Z_{\sigma}$-space. The proof is completed.

Let us note a relative version of [13, Corollary 2.7].

Remark 5.8. Let $X_{n} \in \mathscr{M}$ be a noncompact absolute retract and let $Y_{n}$ be a subset of $X_{n}$ such that $X_{n} \backslash Y_{n}$ is locally homotopy negligible in $X_{n}, n=$ $1,2, \ldots$. If each $Y_{n}$ is an absolute $F_{\sigma \delta}$-set and infinitely many of the $Y_{n}$ 's are $Z_{\sigma}$-spaces, then the pairs $\left(\prod_{n=1}^{\infty} X_{n}, \prod_{n=1}^{\infty} Y_{n}\right)$ and $\left(s, \Omega_{2}\right)$ are homeomorphic. Apply 2.2 together with 3.6. To produce a closed embedding $h: K \rightarrow \prod_{n=1}^{\infty} X_{n}$ 
with $h^{-1}\left(\prod_{n=1}^{\infty} Y_{n}\right)=L$ employ Lemma 5.2 and the fact that $\prod_{n=1}^{\infty} X_{n}$ contains a closed copy of $[0, \infty)$ that lives in $\prod_{n=1}^{\infty} Y_{n}$. Moreover, adopting Theorem 2.2 and Lemma 5.2 to the triple case one can get a homeomorphism of suitable triples (see [9]); 4.8 .

Let us recall that by $L^{p}[a, b]$ we denote the space of equivalence classes of Lebesgue measurable functions $x:[a, b] \rightarrow R$ with

$$
\|x\|_{p}=\left(\int_{a}^{b}|x(t)|^{p} d t\right)^{\min \left(1, \frac{1}{p}\right)}<\infty
$$

with the topology induced by the $F$-norm $\|\cdot\|_{p}, 0<p<\infty$. Write $\tilde{L}^{p}[a, b]=$ $\bigcap_{p^{\prime}<p} L_{p^{\prime}}[a, b], 0<p \leq \infty$, and by $\widetilde{L}_{q}^{p}[a, b]$ denote the set $\widetilde{L}^{p}[a, b]$ with the $\|\cdot\|_{q}$-topology, $q<p$. Note that $\widetilde{L}_{q}^{p}[a, b]$ is dense in $L^{q}[a, b]$. We skip the symbol $[a, b]$ if $[a, b]=[0,1]$.

Theorem 5.9. The pairs $\left(L^{q}, \tilde{L}_{q}^{p}\right)$ and $\left(s, \Omega_{2}\right)$ are homeomorphic for $0<q<$ $p \leq \infty$.

Proof. Mazur's homeomorphism [2, p. 207] of $L^{1}$ onto $L^{q}$ transforms $\widetilde{L}^{p}$ onto $\widetilde{L}^{p q}$. Therefore, it sufficies to consider the case of $q=1$ (and arbitrary $p>1$ ).

We write $\widetilde{L}^{p}=\widetilde{L}_{1}^{p}$. Since $L^{1}$ is a copy of $l^{2}$ [2], it is enough to verify conditions (i)-(iv) of 2.2. The local homotopy negligibility of $L^{1} \backslash \widetilde{L}^{p}$ follows in a standard way. Note that each $L^{p}$ is an $F_{\sigma}$-subspace of $L^{q}$ for $p>q$. (This is a consequence of the facts that $L^{p}$ is an $F_{\sigma}$-subspace of $L^{0}$, the space of measurable functions with the convergence in measure topology (see [13]), and that the $L^{0}$-topology is weaker than the $\|\cdot\|_{q}$-topology.) Select an increasing sequence $\left\{p_{n}\right\}_{n=1}^{\infty} \subset(1, p)$ that converges to $p$. Since $\widetilde{L}^{p}=\bigcap_{n=1}^{\infty} L^{p_{n}}$, we get $\tilde{L}^{p} \in \mathscr{M}_{2}$.

To prove that $\widetilde{L}^{p}$ is a $Z_{\sigma}$-space, we choose $1<p^{\prime}<p$ and write

$$
B_{p^{\prime}}(\varepsilon)=\left\{x \in L^{p^{\prime}}:\|x\|_{p^{\prime}} \leq \varepsilon\right\} .
$$

Since $\widetilde{L}^{p}=\bigcup_{k=1}^{\infty} B_{p^{\prime}}(k) \cap \widetilde{L}^{p}$ it suffices to check that each $A=B_{p^{\prime}}(k) \cap \widetilde{L}^{p}$ is a $Z$-set in $\widetilde{L}^{p}$. First of all, note that $B_{p^{\prime}}(k)$ is a $Z$-set in $L^{1}$ because it is a closed subset of a locally homotopy negligible set $L^{p^{\prime}}$ in $L^{1}$. Then, using the fact that $L^{1} \backslash \widetilde{L}^{p}$ is locally homotopy negligible in $L^{1}$, we infer that $A$ is a $Z$-set in $\widetilde{L}^{p}$ (see [5, Lemma 2.6]).

We make use of 3.1 to verify the strong $\left(\mathscr{M}, \mathscr{M}_{2}\right)$-universality of $\left(L^{1}, \widetilde{L}^{p}\right)$. The map $\Psi$ given by

$$
\Psi(x)=\left(x \mid\left[2^{-n}, 2^{-n+1}\right]\right)_{n=1}^{\infty},
$$

$x \in L^{1}$, is a linear isomorphism of $L^{1}$ onto $\prod_{l^{1}} L^{1}\left[2^{-n}, 2^{-n+1}\right]$. Writing $Z=\Psi\left(\widetilde{L}^{p}\right)$, we have

$$
Z \cap \sum_{l^{1}} L^{1}\left[2^{-n}, 2^{-n+1}\right]=\sum_{l^{1}} \tilde{L}^{p}\left[2^{-n}, 2^{-n+1}\right] .
$$

Since the pair $\left(E_{n}, H_{n}\right)=\left(L^{1}\left[2^{-n}, 2^{-n+1}\right], \widetilde{L}^{p}\left[2^{-n}, 2^{-n+1}\right]\right)$ is (naturally) isomorphic to $\left(L^{1}, \widetilde{L}^{p}\right)$, the lemma below verifies the hypothesis of 3.1 and thus finishes the proof of 5.9 . 
Lemma 5.10. Let $(K, L) \in\left(\mathscr{M}, \mathscr{M}_{2}\right)$. There exists a bounded closed embedding $\psi: K \rightarrow L^{1}$ with $\psi^{-1}\left(\widetilde{L}^{p}\right)=L$.

Proof. We repeat a reasoning from the proof of 5.1. First, we find a bounded closed embedding $\psi^{1}: K \rightarrow L^{1}\left[\frac{1}{2}, 1\right]$ with $\psi^{1}(K) \subset \widetilde{L}^{p}$. Then, we produce a bounded map $\psi^{2}: K \rightarrow L^{1}\left[0, \frac{1}{2}\right]$ with $\left(\psi^{2}\right)^{-1}\left(\widetilde{L}^{p}\left[0, \frac{1}{2}\right]\right)=L$. Finally, we let $\psi=\left(\psi^{1}, \psi^{2}\right)$. To get $\psi^{1}$, we apply 5.3 with $H_{n}=L^{p}\left[\frac{1}{2}, 1\right]$, $E_{n}=L^{1}\left[\frac{1}{2}, 1\right]$, and $C=l^{1}$. It is clear that $H_{n}$ is noncompactly embedded in $E_{n}$. Consequently there exists a bounded closed embedding $\psi^{1}: K \rightarrow$ $\prod_{l^{1}} L^{1}\left[\frac{1}{2}, 1\right]=L^{1}\left[\frac{1}{2}, 1\right]$ such that $\psi^{1}(K) \subset \prod_{l^{1}} L^{p}\left[\frac{1}{2}, 1\right] \subset \prod_{l^{p}} L^{p}\left[\frac{1}{2}, 1\right]=$ $L^{p}\left[\frac{1}{2}, 1\right] \subset \widetilde{L}^{p}\left[\frac{1}{2}, 1\right]$. To obtain $\psi^{2}$, embed $K$ into $I^{\infty}$ and represent $L=$ $\bigcap_{n=2}^{\infty} L_{n}$ with each $L_{n} \quad \sigma$-compact and $L_{n+1} \subset L_{n}$ for $n \geq 2$. Recall that $\left\{p_{n}\right\} \subset(1, p)$ converges to $p$. If we find maps $\varphi_{n}: K \rightarrow L^{p_{n}}\left[2^{-n}, 2^{-n+1}\right]$ such that $\varphi_{n}^{-1}\left(\widetilde{L}^{p}\left[2^{-n}, 2^{-n+1}\right]\right)=L_{n}$ and $\left\|\varphi_{n}(x)\right\|_{p_{n}} \leq 2^{-n}$ for all $x \in K$ and $n \geq 2$, then $\psi^{2}$ defined by

$$
\psi^{2}(x) \mid\left[2^{-n}, 2^{-n+1}\right]=\varphi_{n}(x),
$$

$x \in K, n \geq 2$, is as required.

To produce $\varphi_{n}$, we apply 5.2 for $(X, Y)=\left(B_{p_{n}}\left(2^{-n}\right), B_{p_{n}}\left(2^{-n}\right) \cap \widetilde{L}^{p}\right)$. Since $Y$ is convex and dense in $X, X \backslash Y$ is locally homotopy negligible in $X$. Pick $p_{n}<p^{\prime}<p$. We have

$$
Y=B_{p_{n}}\left(2^{-n}\right) \cap \widetilde{L}^{p}=\bigcup_{k=1}^{\infty} B_{p^{\prime}}(k) \cap B_{p_{n}}\left(2^{-n}\right) \cap \widetilde{L}^{p} .
$$

We claim that each $A=B_{p^{\prime}}(k) \cap B_{p_{n}}\left(2^{-n}\right) \cap \widetilde{L}^{p}$ is a $Z$-set in $Y$. Since $B_{p^{\prime}}(k)$ is a $Z$-set in $L^{p_{n}}$, it easily follows that $B_{p^{\prime}}(k) \cap B_{p_{n}}\left(2^{-n}\right)$ is a $Z$-set in $B_{p_{n}}\left(2^{-n}\right)$. Now the local homotopy negligibility of $X \backslash Y$ in $X$ implies, via [5, Lemma 2.6], that $A$ is a $Z$-set in $Y$. This finishes the proof.

Remark 5.11. One could likely elaborate an abstract scheme of identifying some normed coordinate products that are homeomorphic to $\Omega_{2}$, as done for cartesian products in [13]. Due to replacing the convex structure by a suitable equiconnected structure on $L^{0}([0,1], G)$, the space of measurable $G$ valued functions on $[0,1]$, it was proved in [13] that $\widetilde{L}^{p}([0,1], G)$ (with the $L^{0}$-topology) is homeomorphic to $\Omega_{2}$, provided $G$ is a closed unbounded subset of a Banach space. Using 3.6 and 5.2, one can show that the pair $\left(L^{0}([0,1], G), \widetilde{L}^{p}([0,1], G)\right)$ is homeomorphic to $\left(s, \Omega_{2}\right)$ for $0<p \leq \infty$. To produce a closed embedding of $R^{\infty}$ in $L^{0}([0,1], G)$ with values in $B=$ $\left\{x \in L^{1}:|x(t)| \leq \varepsilon\right.$ almost everywhere $\}$, we use the argument of 5.3 and the fact that $B \cap L^{0}([0,1], G)$ is a copy of $l^{2}$ [2]. It is likely that the pairs $\left(L^{q}([0,1], G), \widetilde{L}_{q}^{p}([0,1], G)\right)$ and $\left(s, \Omega_{2}\right)$ are also homeomorphic.

By $l^{p}$ we denote the space of real-valued sequences $x=\left(x_{n}\right)$ such that

$$
\|x\|_{p}=\left(\sum_{n=1}^{\infty}\left|x_{n}\right|^{p}\right)^{\min \left(1, \frac{1}{p}\right)}<\infty
$$

with the topology induced by the $F$-norm $\|\cdot\|_{p}, 0<p<\infty$. Write $\widetilde{l}^{p}=$ 
$\bigcap_{p^{\prime}>p} l^{p^{\prime}}, 0 \leq p<\infty$, and denote by $\widetilde{l}_{q}^{p}$ the space $\widetilde{l}^{p}$ with the $\|\cdot\|_{q}$-topology, $q>p$. Note that $\widetilde{l}_{q}^{p}$ is a dense linear subspace of $l^{q}$.

Theorem 5.12. The pairs $\left(l^{q}, \tilde{l}_{q}^{p}\right)$ and $\left(s, \Omega_{2}\right)$ are homeomorphic for $0 \leq p<$ $q<\infty$.

Proof. As in the proof of 5.9 , we only need to check that $\left(l^{1}, \widetilde{l}^{p}\right), 0<p<1$, fulfils conditions (i)-(iv) of 2.2 ; we write $\widetilde{l}^{p}=\widetilde{l}_{1}^{p}$. A verification of (i) and (iii) is almost the same as in 5.9 and uses the observation that

$$
B_{p^{\prime}}(\varepsilon)=\left\{x \in l^{p^{\prime}}:\|x\| \leq \varepsilon\right\}
$$

is closed in the $\|\cdot\|_{p}$-topology $\left(p>p^{\prime}\right)$. Also, every set $B_{p^{\prime}}(k) \cap \widetilde{l}^{p}$ is a $Z$-set in $\widetilde{l}^{p}$, yielding (ii). To verify (iv) we make use of 3.1. Decompose $N$ into pairwise disjoint infinite sets $N_{1}, N_{2}, \ldots$. Consider the linear isomorphism $\Psi: l^{1} \rightarrow \prod_{l^{1}} l^{1}\left(N_{n}\right)$, where $l^{1}\left(N_{n}\right)$ is an isomorphic copy of $l^{1}$ of sequences indexed by integers of $N_{n}$, given by

$$
\Psi(x)=\left(\left(x_{k}\right)_{k \in N_{1}},\left(x_{k}\right)_{k \in N_{2}}, \ldots\right),
$$

$x \in l^{1}$. Writing $Z=\Psi\left(\widetilde{l^{p}}\right)$, we see that

$$
Z \cap \sum_{l^{1}} l^{1}\left(N_{n}\right)=\sum_{l^{1}} \widetilde{l}^{p}\left(N_{n}\right)
$$

The following lemma enables us to apply 3.1 and hence to finish the proof of 5.12 .

Lemma 5.13. Let $(K, L) \in\left(\mathscr{M}, \mathscr{M}_{2}\right)$. There exists a bounded closed embedding $\psi: K \rightarrow l^{1}$ with $\psi^{-1}\left(\widetilde{l}^{p}\right)=L$.

Proof. We follow the proof of 5.10. As in 5.10 we embed $K$ into $I^{\infty}$, represent $L=\bigcap_{n=1}^{\infty} L_{n}$, and pick a sequence $\left(p_{n}\right) \subset(p, 1)$ convergent to $p$. A bounded closed embedding $\psi^{1}: K \rightarrow l^{1}\left(N_{1}\right)$ with $\psi^{1}(K) \subset \widetilde{l}^{p}$ is obtained via Lemma 5.3. We take $H_{n}=\left(l^{p}\left(N_{1}\right),\|\cdot\|_{p}\right), E_{n}=l^{1}\left(N_{1}\right)$, and $C=l^{1}$. (Formally, we are not eligible to apply 5.3 because $H_{n}$ is not a normed space. This assumption was only used to construct the closed embedding of $[0, \infty)$. In our case the unit closed ball $B$ in $l^{p}\left(N_{1}\right)$ is homeomorphic, via Mazur's homeomorphism [2, p. 207], to the closed unit ball in the Hilbert space which, in turn, is homeomorphic to $R^{\infty}$. Therefore $B$ being closed in $l^{1}\left(N_{1}\right)$ admits a required embedding.) Hence, we get a bounded closed embedding $\psi^{1}: K \rightarrow \prod_{l^{1}} l^{1}\left(N_{1}\right)=l^{1}\left(N_{1}\right)$ with $\psi^{1}(K) \subset \prod_{l^{1}} l^{p}=l^{p} \subset \widetilde{l^{p}}$. To produce $\psi^{2}$, we apply 5.2 to the pair $(X, Y)=\left(B_{p_{n}}\left(2^{-n}\right), B_{p_{n}}\left(2^{-n}\right) \cap \tilde{l}^{p}\right)$ and find maps $\varphi_{n}: K \rightarrow l^{p_{n}}\left(N_{n}\right), n \geq 2$, with $\varphi_{n}^{-1}\left(\widetilde{l}^{p}\left(N_{n}\right)\right)=L_{n}$ and $\left\|\varphi_{n}(x)\right\|_{p_{n}} \leq 2^{-n}$ for all $x \in K$. It is easy to see that the map $\psi^{2}(x)=\left(\varphi_{n}(x)\right)_{n=2}^{\infty}$ satisfies $\left(\psi^{2}\right)^{-1}\left(\tilde{l}^{p}\left(N \backslash N_{1}\right)\right)=L$. We let $\psi=\left(\psi^{1}, \psi^{2}\right)$.

Let us formulate a more specific result concerning $\tilde{l}^{p}$-products whose proof is a modification of the proof of 5.7 (and therefore will be omitted).

Theorem 5.14. Let, for $n \geq 1, X_{n}$ be a subset of a Banach space $\left(E_{n},\|\cdot\|_{n}\right)$ with $0 \in X_{n}$ so that $\inf _{n \geq 1} \operatorname{diam}\left(X_{n}\right)=\alpha>0$. Assume that each $X_{n}$ is an 
absolute retract that is an absolute $F_{\sigma \delta}$-set. Then the space

$$
\tilde{l}^{p}\left(X_{n}\right)=\left\{\left(x_{n}\right) \in \prod_{n=1}^{\infty} X_{n}: \forall_{p^{\prime}>p} \sum_{n=1}^{\infty}\left\|x_{n}\right\|_{n}^{p^{\prime}}<\infty\right\}
$$

(as a subspace of $\prod_{n=1}^{\infty} X_{n}$ ) is homeomorphic to $\Omega_{2}$ for every $0 \leq p<\infty$.

Remark 5.15. Assume that each $X_{n} \in \mathscr{M}$. We may ask whether $\left(\prod_{n=1}^{\infty} X_{n}\right.$, $\left.\tilde{l}^{p}\left(X_{n}\right)\right)$ is homeomorphic to $\left(s, \Omega_{2}\right)$. This, in general, is not necessarily the case. The space $\widetilde{l}^{p}(R)=\widetilde{l}^{p}$ is contained in a $\sigma$-compact subset of $R^{\infty}$ (cf. Remark 5.6). Let us notice that the pair $\left(R^{\infty}, \widetilde{l}^{p}\right)$ is strongly $\left(\mathscr{M}_{0}, \mathscr{M}_{2}\right)$-universal. The assertion of Theorem 2.2 holds if one replaces $\mathscr{M}$ by $\mathscr{M}_{0}$ and add in the hypothesis that both $Y_{1}$ and $Y_{2}$ are contained in $\sigma$-compact subsets of $X$. As a consequence, the pairs $\left(R^{\infty}, c_{0}\right)$ and $\left(R^{\infty}, \widetilde{l}^{p}\right)$ are homeomorphic for $0 \leq p<\infty$. This shows that two $\mathscr{L}$-absorbing sets $Y_{1}$ and $Y_{2}$ can be relatively homeomorphic in a copy $X$ of $l^{2}$ while none of the pairs $\left(X, Y_{1}\right)$ and $\left(X, Y_{2}\right)$ are strongly $(\mathscr{M}, \mathscr{L})$-universal.

\section{The SPACES $F_{\alpha}$ AND $G_{\alpha}$ AS FACTORS OF EXOTIC PRE-HiLbeRT SPACES}

In this section we present some examples concerning the topological classification of pre-Hilbert spaces. Examples we deal with are of the form $Y(A) \times F_{\alpha}$ and $Y(A) \times G_{\alpha}$, where $Y(A)=\operatorname{span}(A)$ and $A$ is a linearly independent subset of $l^{2}$.

Fix a linearly independent arc $T=[0,1]$ in $l^{2}$ such that $Y(A)$ is dense in $l^{2}$ for every infinite set $A \subseteq T$ (see [2, p. 267]). Since $Y(A)$ is contained in a $\sigma$-compact subspace of $l^{2}, Y(A)$ is a $Z_{\sigma}$-space provided it is infinitedimensional (i.e., $A$ is infinite).

Proposition 6.1. Let $A$ be any subset of $T$ and $\alpha \geq 1$. Then:

(a) $Y(A) \times F_{\alpha}$ (respectively, $\left.Y(A) \times G_{\alpha}\right)$ contains no closed copy of $l^{2} \backslash G_{\alpha+1}$ (respectively, $\left.l^{2} \backslash F_{\alpha+1}\right)$,

(b) $Y(A) \times F_{\alpha}\left(\right.$ respectively, $\left.Y(A) \times G_{\alpha}\right)$ contains no closed copy of $l^{2} \backslash F_{\alpha}$ (respectively, $l^{2} \backslash G_{\alpha}$ ).

First, in full detail, we consider the following particular case of part (b) with $\alpha=1$ (as $l^{2} \backslash F_{1}$ is a copy of $l^{2}$, see [2]).

Lemma 6.2. For every subset $A$ of $T$, the space $Y(A) \times \Sigma$ contains no closed copy of $l^{2}$. In particular, $Y(A) \times \Sigma$ is homeomorphic neither to $\Lambda_{\alpha}, \alpha \geq 2$, nor to $\Omega_{\alpha}, \alpha \geq 1$.

Proof. We apply the cross-section argument described in 4.3. For $k$ and $p \geq 1$, we write

$$
C_{k}^{p}=\left\{\left(t_{1}, t_{2}, \ldots, t_{k}\right) \in T^{k}: t_{1} \leq t_{2} \leq \cdots \leq t_{k},\left\|t_{i}-t_{j}\right\| \geq \frac{1}{p}\right\}
$$

and

$$
D_{k}^{p}=\left\{\left(\lambda_{1}, \lambda_{2}, \ldots, \lambda_{k}\right) \in R^{k}: \frac{1}{p} \leq\left|\lambda_{i}\right| \leq p \text { for all } i\right\} .
$$

(The union $\bigcup_{k, p=1}^{\infty} C_{k}^{p}$ is a particular $\sigma$-compact cross-section for $T^{k}$.) The map $\chi_{k}$ given by $\chi_{k}\left(\left(t_{1}, t_{2}, \ldots, t_{k}\right),\left(\lambda_{1}, \lambda_{2}, \ldots, \lambda_{k}\right)\right)=\lambda_{1} t_{1}+\lambda_{2} t_{2}+\cdots+\lambda_{k} t_{k}$ 
is a homeomorphism of $C_{k}^{p} \times D_{k}^{p}$ onto $M_{k}^{p} \subset Y(T)$. Clearly,

$$
M_{k}^{p} \cap Y(A)=\chi_{k}\left(\left(C_{k}^{p} \cap A^{k}\right) \times D_{k}^{p}\right)=N_{k}^{p}
$$

is a closed subset in $Y(A)$. Since $Y(A)=\{0\} \cup \bigcup_{k, p=1}^{\infty} N_{k}^{p}$, we get

$$
Y(A) \times \Sigma=(\{0\} \times \Sigma) \cup \bigcup_{k, p=1}^{\infty}\left(N_{k}^{p} \times \Sigma\right) .
$$

Assume that $X$ being a copy of $l^{2}$ is contained as a closed subset of $Y(A) \times \Sigma$. Using a Baire category argument and the fact that no open set in $l^{2}$ is $\sigma$ compact we find indices $k$ and $p$ such that $N_{k}^{p} \times \Sigma$ contains an open subset $U$ of $X$. It follows that a copy $B$ of a closed ball in $l^{2}$ inscribed in $U$ is closed in $N_{k}^{p} \times \Sigma$. It is easy to see that there exists a connected set $K \subset A^{k}$ such that

$$
B \subset \chi_{k}\left(\left(K \cap C_{k}^{p}\right) \times D_{k}^{p}\right) \times \Sigma
$$

Each connected subset $K$ of $A^{k}$ is of the form $I_{1} \times I_{2} \times \cdots \times I_{k}$, where every $I_{j}$ is a connected component of $A$ (i.e., $I_{j}$ is an interval). Hence, $K \cap C_{k}^{p}$ is locally compact and so is $\chi_{k}\left(\left(K \cap C_{k}^{p}\right) \times D_{k}^{p}\right)$. Finally, $B$, being a closed subset of a $\sigma$-compact space $\chi_{k}\left(\left(K \cap C_{k}^{p}\right) \times D_{k}^{p}\right) \times \Sigma$, is itself $\sigma$-compact, a contradiction.

Proof of 6.1. Assume $Y(A) \times F_{\alpha}$ contains a closed copy $X$ of $l^{2} \backslash G_{\alpha+1}$. Using the notation of the proof of 6.2 , we have

$$
Y(A) \times F_{\alpha}=\left(\{0\} \times F_{\alpha}\right) \cup \bigcup_{k, p=1}^{\infty}\left(N_{k}^{p} \times F_{\alpha}\right) .
$$

Since $X$ is a Baire space (see 4.9), there exist $k$ and $p$ and a closed set $P \subset N_{k}^{p} \times F_{\alpha}$ such that $P$ has nonempty interior in $X$ and $P$ is a copy of $B \backslash G_{\alpha+1}$ for some closed ball in $l^{2}$. According to 4.9, $P$ is connected. As in the proof of 6.2 , we get

$$
P \subset \chi_{k}\left(\left(K \cap C_{k}^{p}\right) \times D_{k}^{p}\right) \times F_{\alpha},
$$

where $K \cap C_{k}^{p}$ is locally compact. Now, it follows that $\chi_{k}\left(\left(K \cap C_{k}^{p}\right) \times D_{k}^{p}\right) \times F_{\alpha} \in$ $\mathscr{A}_{\alpha}$; consequently $P \in \mathscr{A}_{\alpha}$. Since $X$ is homogeneous (see 4.9) and the interior of $P$ in $X$ is nonempty, $X$ is locally in the class $\mathscr{A}_{\alpha}$. The latter yields $X \in \mathscr{A}_{\alpha}$, contradicting $G_{\alpha+1} \in \mathscr{M}_{\alpha+1} \backslash \mathscr{A}_{\alpha+1}$.

All the remaining cases can be proved in the same way. (A minor change is needed for $G_{1}$; namely, $G_{1}$ must be represented as a countable union of complete metrizable spaces.)

Corollary 6.3. For every subset $A$ of $T$ the spaces $Y(A) \times F_{\alpha}$ and $Y(A) \times G_{\alpha}$, $\alpha \geq 1$, are topologically distinct.

Proof. By 6.1, $Y(A) \times F_{\alpha}$ contains no closed copy of $l^{2} \backslash F_{\alpha} \in \mathscr{M}_{\alpha}$. Since $\mathscr{M}_{\alpha} \subset \mathscr{A}_{\beta} \cap \mathscr{M}_{\beta}$ for $\beta>\alpha$, the spaces $Y(A) \times F_{\beta}$ and $Y(A) \times G_{\beta}$ do contain such a copy. Also $Y(A) \times G_{\alpha}$ contains a closed copy of $l^{2} \backslash F_{\alpha}$. As a consequence, we conclude that $Y(A) \times F_{\alpha}$ is not homeomorphic to $Y(A) \times F_{\beta}$ for $\alpha \neq \beta$ and that $Y(A) \times F_{\alpha}$ is not homeomorphic to $Y(A) \times G_{\beta}$ for $\beta \geq \alpha$. Analogously, we prove that $Y(A) \times G_{\alpha}$ is not homeomorphic to $Y(A) \times G_{\beta}$ for $\beta \neq \alpha$ and that $Y(A) \times G_{\beta}$ is not homeomorphic to $Y(A) \times F_{\alpha}$ for $\beta \leq \alpha$.

The same argument applies in the following 
Corollary 6.4. For every subset $A$ of $T$, the spaces $Y(A) \times F_{\alpha}$ and $Y(A) \times G_{\alpha}$ are homeomorphic neither to $F_{\beta}$ nor to $G_{\beta}$ for $\beta \neq \alpha$.

Corollary 6.5. We have:

(a) if $A \in \mathscr{A}_{\alpha} \backslash \mathscr{M}_{\alpha}$, then the spaces $F_{\alpha}, Y(A) \times F_{\beta}$, and $Y(A) \times G_{\beta}$ belong to $\mathscr{A}_{\alpha} \backslash \mathscr{M}_{\alpha}$ and are topologically distinct for $\beta<\alpha$,

(b) if $A \in \mathscr{M}_{\alpha} \backslash \mathscr{A}_{\alpha}$, then the spaces $G_{\alpha}, Y(A) \times G_{\beta}$, and $Y(A) \times F_{\beta}$ belong to $\mathscr{M}_{\alpha} \backslash \mathscr{A}_{\alpha}$ and are topologically distinct for $\beta<\alpha$,

(c) if $A \in \mathscr{P}_{n} \backslash \bigcup_{k<n} \mathscr{P}_{k}$, then the spaces $Y(A) \times F_{\alpha}$ and $Y(A) \times G_{\alpha}$ belong to $\mathscr{P}_{n} \backslash \bigcup_{k<n} \mathscr{P}_{k}$ and are topologically distinct,

(d) if $A \notin \bigcup_{n=1}^{\infty} \mathscr{P}_{n}$, then the spaces $Y(A) \times F_{\alpha}$ and $Y(A) \times G_{\alpha}$ do not belong to $\bigcup_{n=1}^{\infty} \mathscr{P}_{n}$ and are topologically distinct.

Corollary 6.5 is a direct consequence of 6.3 and 6.4 and the following fact, which seems to be well known; however we could not find it formulated in such a generality in literature.

Lemma 6.6. We have:

(a) if $A \in \mathscr{A}_{\alpha} \backslash \mathscr{M}_{\alpha}$, then $Y(A) \in \mathscr{A}_{\alpha} \backslash \mathscr{M}_{\alpha}, \alpha \geq 1$,

(b) if $A \in \mathscr{M}_{\alpha} \backslash \mathscr{A}_{\alpha}$, then $Y(A) \in \mathscr{M}_{\alpha} \backslash \mathscr{A}_{\alpha}, \alpha \geq 2$,

(c) if $A \in \mathscr{P}_{n} \backslash \bigcup_{k<n} \mathscr{P}_{k}$, then $Y(A) \in \mathscr{P}_{n} \backslash \bigcup_{k<n} \mathscr{P}_{k}, n \geq 1$,

(d) if $A \notin \bigcup_{n=1}^{\infty} \mathscr{P}_{n}$, then $Y(A) \notin \bigcup_{n=1}^{\infty} \mathscr{P}_{n}$.

Proof. Since $A$ is closed in $Y(A), A \notin \mathscr{L}$ implies $Y(A) \notin \mathscr{L}$ provided $\mathscr{L}$ is closed with respect to closed subsets. Therefore, it suffices to show that $A \in \mathscr{L}$ implies $Y(A) \in \mathscr{L}$, where $\mathscr{L}=\mathscr{A}_{\alpha}, \mathscr{M}_{\alpha}$ and $\bigcup_{k<n} \mathscr{P}_{k}$. The case $\mathscr{A}_{\alpha}$ and $\bigcup_{k<n} \mathscr{P}_{n}$ is a result of Klee [2, p. 272]. Let $A \in \mathscr{M}_{\alpha}$ and $\alpha \geq 2$. Represent $A=\bigcap_{n=1}^{\infty} A_{n}, A_{n} \in \mathscr{A}_{\beta_{n}}$ for $\beta_{n}<\alpha$, and employ $Y\left(A_{n}\right) \in \mathscr{A}_{\beta_{n}}$ to conclude that $Y(A)=\bigcap_{n=1}^{\infty} Y\left(A_{n}\right) \in \mathscr{M}_{\alpha}$.

Remark 6.7. Corollary 6.5(a) and (b) (see also 6.2) provide a negative answer to the question of whether a pre-Hilbert space that contains a Hilbert cube and is of the exact Borelian class of order $\alpha$ must be homeomorphic to either $F_{\alpha}$ or $G_{\alpha}, \alpha \geq 2$. The answer to this question is "yes" for $\mathscr{A}_{1}$.

Remark 6.8. From 6.5(c) it follows that each class $\mathscr{P}_{n} \backslash \bigcup_{k<n} \mathscr{P}_{k}$ contains uncountably many topologically distinct pre-Hilbert spaces that are $Z_{\sigma}$-spaces.

Remark 6.9. Each class $\mathscr{A}_{\alpha} \backslash \mathscr{M}_{\alpha}, \alpha \geq 1$, and $\mathscr{M}_{\alpha} \backslash \mathscr{A}_{\alpha}, \alpha \geq 2$, contains uncountably many topologically distinct pre-Hilbert spaces that are $Z_{\sigma}$-spaces. To show this, take $A \in \mathscr{A}_{\alpha} \backslash \mathscr{M}_{\alpha}$ (respectively, $A \in \mathscr{M}_{\alpha} \backslash \mathscr{A}_{\alpha}$ ) and repeat Henderson and Petczyński's argument to the spaces $Y(A) \times X, X \in \mathscr{X}$, where $\mathscr{X}$ is that of $[2$, p. 282].

\section{REFERENCES}

1. C. Bessaga, On the topological classification of complete linear metric spaces, Fund. Math. 56 (1965), 251-288.

2. C. Bessaga and A. Pełczyński, Selected topics in infinite-dimensional topology, PWN, Warsaw, 1975.

3. M. Bestvina and J. Mogilski, Linear maps do not preserve countable-dimensionality, Proc. Amer. Math. Soc. 93 (1985), 661-666. 
4. __ Characterizing certain incomplete infinite-dimensional retracts, Michigan Math. J. 33 (1986), 291-313.

5. R. Cauty, Caractérisation topologique de l'espace des fonctions dérivables, Fund. Math. 138 (1991), 35-58.

6. L_ Les fonctions continues et les fonctions integrables au sens de Riemann comme sousespaces de $\mathscr{L}^{1}$, Fund. Math. 139 (1991), 23-36.

7. __ Sur deux espaces de fonctions non dérivables, Fund. Math. (to appear).

8. __ Un exemple d'ensembles absorbants non équivalents, Fund. Math. 140 (1991), 49-61.

9. J. J. Dijkstra, J. van Mill, and J. Mogilski, The space of infinite-dimensional compacta and other topological copies of $\left(l_{f}^{2}\right)^{\omega}$, Pacific J. Math. 152 (1992), 255-273.

10. J. J. Dijkstra and J. Mogilski, The topological product structure of systems of Lebesgue spaces, Math. Ann. 290 (1991), 523-527.

11. T. Dobrowolski, S. P. Gul'ko, and J. Mogilski, Function spaces homeomorphic to the countable product of $l_{2}^{f}$, Topology Appl. 34 (1990), 153-160.

12. T. Dobrowolski, W. Marciszewski, and J. Mogilski, On topological classification of function spaces $C_{p}(X)$ of low Borel complexity, Trans. Amer. Math. Soc. 328 (1991), 307-324.

13. T. Dobrowolski and J. Mogilski, Certain sequence and function spaces homeomorphic to the countable product of $l_{f}^{2}$, J. London Math. Soc. (to appear).

14. V. Klee, Some topological properties of convex sets, Trans. Amer. Math. Soc. 78 (1955), 30-45.

15. S. Mazur and L. Sternbach, Über die Borelschen Typen von linearen Mengen, Studia Math. 4 (1933), 48-55.

16. H. Torunczyk, On Cartesian factors and the topological classification of linear metric spaces, Fund. Math. 88 (1975), 71-87.

17. _ Concerning locally homotopy negligible sets and characterization of $l_{2}$-manifolds, Fund. Math. 101 (1978), 93-110.

18. __ Characterizing Hilbert space topology, Fund. Math. 111 (1981), 247-262.

Universite Paris Vi, Analyse Complexe et Géometrie, 4, Place Jussieu, 75252 ParisCedex 05, France

InStytut Matematyki, Uniwersytet WaRsZawski, PKIN IXP., 00-901 WarsZaWa, Poland 73019

Current address: Department of Mathematics, University of Oklahoma, Norman, Oklahoma

E-mail address: tdobrowo@nsfuvax.math.uoknor.edu 